Florida International University FIU Digital Commons

$11-18-1994$

\title{
Improved frequency response measurements in the human visual system
}

Yuping Chen

Florida International University

DOI: $10.25148 /$ etd.FI14060183

Follow this and additional works at: https://digitalcommons.fiu.edu/etd

Part of the Physics Commons

\section{Recommended Citation}

Chen, Yuping, "Improved frequency response measurements in the human visual system" (1994). FIU Electronic Theses and Dissertations. 2151.

https://digitalcommons.fiu.edu/etd/2151 
FLORIDA INTERNATIONAL UNIVERSITY

$$
\text { Miami, Florida }
$$

\title{
Improved Frequency Response Measurements in the Human Visual system
}

\begin{abstract}
A thesis submitted in partial satisfaction of the requirements for the degree of Master of Science in Physics
\end{abstract}

by

Yuping Chen 
To: Dr. Arthur W. Herriott, Dean

College of Arts and Sciences

This thesis, written by Yuping chen, and entitled Improved Frequency Response Measurements in the Human Visual system, having been approved in respect to style and intellectual content, is referred to you for judgement.

We have read the thesis and recommend that it be approved.

Stephan Mintz

Yesim Darici

John T. Landrum

Richard A. Bone, Major Professor

Date of Defense: November 18, 1994

The thesis of Yuping Chen is approved.

Dean Arthur W. Herriott College of Arts and Sciences

Dr. Richard L. Campbell

Dean of Graduate Studies

Florida International University, 1994 


\section{ACKNOWLEDGEMENT}

I am grateful to my advisor, Professor Richard A. Bone, for his continuous support and advice during the process of this study. I have always benefited from discussion with him. It would have been impossible to finish this thesis without his enormous help from the beginning of the research.

I deeply appreciate the help of my committee Professors Stephan L. Mintz, Yesim Darici, and John T. Landrum. They carefully read my manuscript and contributed precious advice.

Finally, I wish to express my gratitude to $\mathrm{Mr}$. Huang and Mr. Orta in the Physics Department for their great help in the accomplishment of this research. 


\title{
ABSTRACT OF THE THESIS
}

\section{Improved Frequency Response Measurements \\ in the Human visual system}

\author{
by \\ Yuping Chen \\ Florida International University \\ Miami, Florida \\ Professor Richard A. Bone, Major Professor
}

The temporal frequency response characteristics of the human visual system have been investigated by combining the increment threshold and sinusoidal flicker methods. Gain and phase spectra were obtained for two subjects using improved equipment and methodology compared with previous investigations. While the output of the system for sinusoidal input stimuli was generally also sinusoidal, as required for a linear system, other non-linearities emerged. Gain spectra for both subjects indicated a low-pass filtering action. Phase spectra showed a possible low frequency phase lead followed by a high frequency lag. Earlier studies suggested that the system might be a minimum phase system and, as such, would allow the phase spectrum to be derived from the gain spectrum via the Kramers-Kronig relations. This possibility was examined in light of the measured gain and phase spectra and found to be implausible. 
CONTENTS

\section{Abstract}

1. Introduction and previous Temporal studies . . . . . 1

1.1 Introduction and overview of previous studies . 1

1.2 Critical flicker frequency method . . . . . . . 6

1.3 Increment threshold method . . . . . . . . 8

1.4 Combined increment threshold and flicker method 11

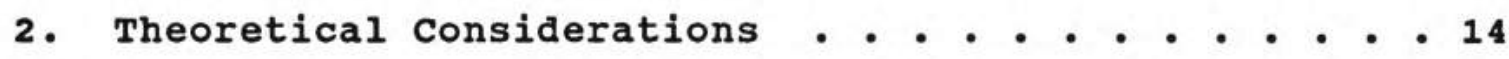

2.1 General theory of linear systems . . . . . . . . 14

2.2 Diffusion model . . . . . . . . . . . . 20

2.3 Relationship between gain and phase . . . . . 23

3. Experiments . - . . . . . . . . . . . . . 26

3.1 Experimental requirements . . . . . . . . . 26

3.2 Experimental system . . . . . . . . . 28

3.3 Adjustments and calibrations . . . . . . . . 34

3.4 Experimental procedures . . . . . . . . . . 38

4. Results, calculations and discussion . . . . . . . 40

4.1 Output waveform . . . . . . . . . . . 40

4.2 Effects of modulation . . . . . . . . . . 52

4.3 Gain characteristics . . . . . . . . . . 56

4.4 Phase shift characteristics . . . . . . . . . 58

4.5 Relationship between gain and phase . . . . . 61

4.6 The impulse response . . . . . . . . . . . 64

5. Conclusions - . . . . . . . . . . . . . 68

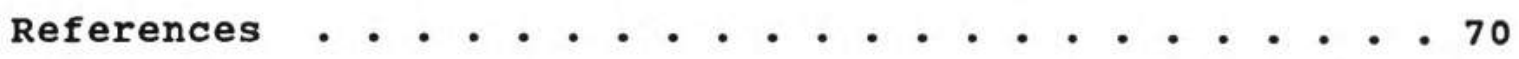




\section{LIST OF FIGURES}

Figure 1.1 Delange C.F.F. curves . . . . . . . . . 4

Figure 1.2 Phase shift calculated by Stork and Falk . . 6

Figure 1.3 Steady response of the visual system . . . . 9

Figure 1.4 Increment threshold for a single

flash response . . . . . . . . . 10

Figure 1.5 Square wave flicker stimulus . . . . . . 11

Figure 1.6 visual field . . . . . . . . . . . . 12

Figure 1.7 Increment threshold at peak and

trough of output . . . . . . . . 13

Figure 2.1 "Black box" model for the a visual system 14

Figure 2.2 Fourier analysis for a linear system . . 15

Figure 2.3 Schematic diagram of the proposed

two-stage model ........... . 22

Figure 3.1 Experiment system . . . . . . . . 28

Figure 3.2 Diagram of stacked beam-splitters . . . . 32

Figure 3.3 Modulation as a function

of orientation of polarizer $\mathrm{P}_{4}$. . . . . 35

Figure 3.4 Relative intensity vs recorder

readings of wedge position . . . . . 37

Figure 4.1 Distorted output waveform . . . . . . . 41

Figure 4.2 True sinusoid and distorted sinusoid . . 41

Figure 4.3 Output waveform at $60 \%$ modulation . . . 43

Figure 4.4 Output waveform at $90 \%$ modulation (Y.P.C.) 47 
Figure 4.5 Output waveform at $90 \%$ modulation (R.A.B.) 50 Figure 4.6 Input modulation . . . . . . . . . 52 Figure 4.7 output amplitude as

a function of modulation ..... . . 53

Figure 4.8 output amplitude vs modulation $(\mathrm{F}=30 \mathrm{~Hz}) 54$ Figure 4.9 Phase shift curves vs modulation . . . 55 Figure 4.10 Gain curves at $60 \%$ modulation . . . . 56 Figure 4.11 Gain curves at 90\% modulation (R.A.B.) . 57 Figure 4.12 Gain curves at 90\% modulation (Y.P.C) . . 57 Figure 4.13 Phase shift curves at $90 \%$ modulation (Y.P.C.) . . . . . . . 58

Figure 4.16 Phase shift curves at $90 \%$ modulation (R.A.B.) . . . . . . . 59

Figure 4.15 Phase shift curves at 90\% modulation . 59 Figure 4.16 Time delay correction for the visual system........... 60

Figure 4.17 Relative gain curve . . . . . . . . 62

Figure 4.18 Phase shift spectrum . . . . . . . 63

Figure 4.19 Impulse response . . . . . . . . . 66

Figure 4.20 Impulse responses of the two-stage model 67 


\section{Chapter 1. Introduction and Previous Temporal Studies}

\subsection{Introduction and overview of previous studies}

The human visual system, consisting principally of the eye, lateral geniculate nucleus and visual cortex, is a highly complex system which detects and processes optical signals. According to current research, different types of visual information are processed and transmitted via different "channels". For example, the "achromatic luminance channel" appears to add signals from the red and green sensitive cones and sends a signal to the brain concerning the brightness of the perceived object. Research in spatial processing, relying largely on the perception of gratings, has revealed the existences of a number of spatial frequency channels. Another area of research is temporal processing, the subject of this thesis.

Temporal processing refers to processing of time-dependent signals, usually visual stimuli whose luminance varies with time. The basic problem is to determine the response of the system for any time varying input. From the theory of linear systems, if the "impulse response", h(t), i.e. the response to an impulse input (a delta function), is known, then the general response $y(t)$ to any other input $x(t)$ is just

$$
y(t)=h(t) \otimes x(t)
$$

In the case of the achromatic channel of the visual system, the output is 
perception of brightness. Considerable effort has gone into trying to measure how this perception changes with time following stimulation by a very brief flash - an impulse input - but the experiment is a difficult one.

An alternative approach, exploited in the present study is to obtain the "frequency response" of the system. This involves determining the gain and phase shift as functions of frequency produced by the system for sinusoidally modulated input stimuli. Once these functions are known, the response to any other periodic input is found as follows:

1. Fourier analyze the input into its sinusoidal components.

2. Determine the amplitude and phase of the sinusoidal output corresponding to each component.

3. Add these outputs to determine the resultant output.

While the gain of the visual system has been determined repeatedly and under a great variety of adaptation conditions, phase measurements are almost completely lacking. The purpose of the present study is to provide accurate phase measurements, as well as gain, over the complete frequency range to which the visual system is sensitive.

It was Ives ${ }^{[1-2]}$ who first introduced the use of visual stimuli varying periodically in radiance as a function of time. Under scotopic conditions (low light levels, rod detection), he found that the "critical fusion frequency" (a frequency above which the sensation of flicker is not produced) was independent of the average intensity, but different for each wave-form of the stimulus. The experimental results may be 
summarized in the empirical formula for the critical fusion frequency, $f$ :

$$
\mathrm{f}=\mathrm{C} \log 2 \mathrm{~W} / \delta
$$

where $\mathrm{W}$ is the coefficient of the first periodic term of the Fourier expansion representing the waveform, divided by the mean intensity of the stimulus; $\mathrm{C}$ and $\delta$ are constants.

Delange ${ }^{[3-6]}$ confirmed the observation using photopic conditions (high light levels cone detection) for a wide variety of waveforms, shown in Fig. 1.1. It was found that with constant average luminance, the points observed for various time functions fit into one smooth curve which for low luminance was monotonic.

The modulation threshold curves of Fig. 1.1 show a peak of sensitivity in the frequency range 5 to $10 \mathrm{~Hz}$, with a gradual decrease of sensitivity at lower frequencies, and a very sharp decrease at higher frequencies. This implies that for the higher frequency harmonics, the gain of the system is essentially zero. Delange also proposed an electrical analog for the system that consisted of several (identical but isolated ) stages of low pass exponential (RC) filtering in cascade. This model was modified by Fuortes and Hodgkin ${ }^{[7]}$ who added time constants and feedback control of the gain for all stages. A number of other investigators carried out these types of experiment using visual stimuli which were sinusoidally modulated in luminance ${ }^{[8-13]}$. 


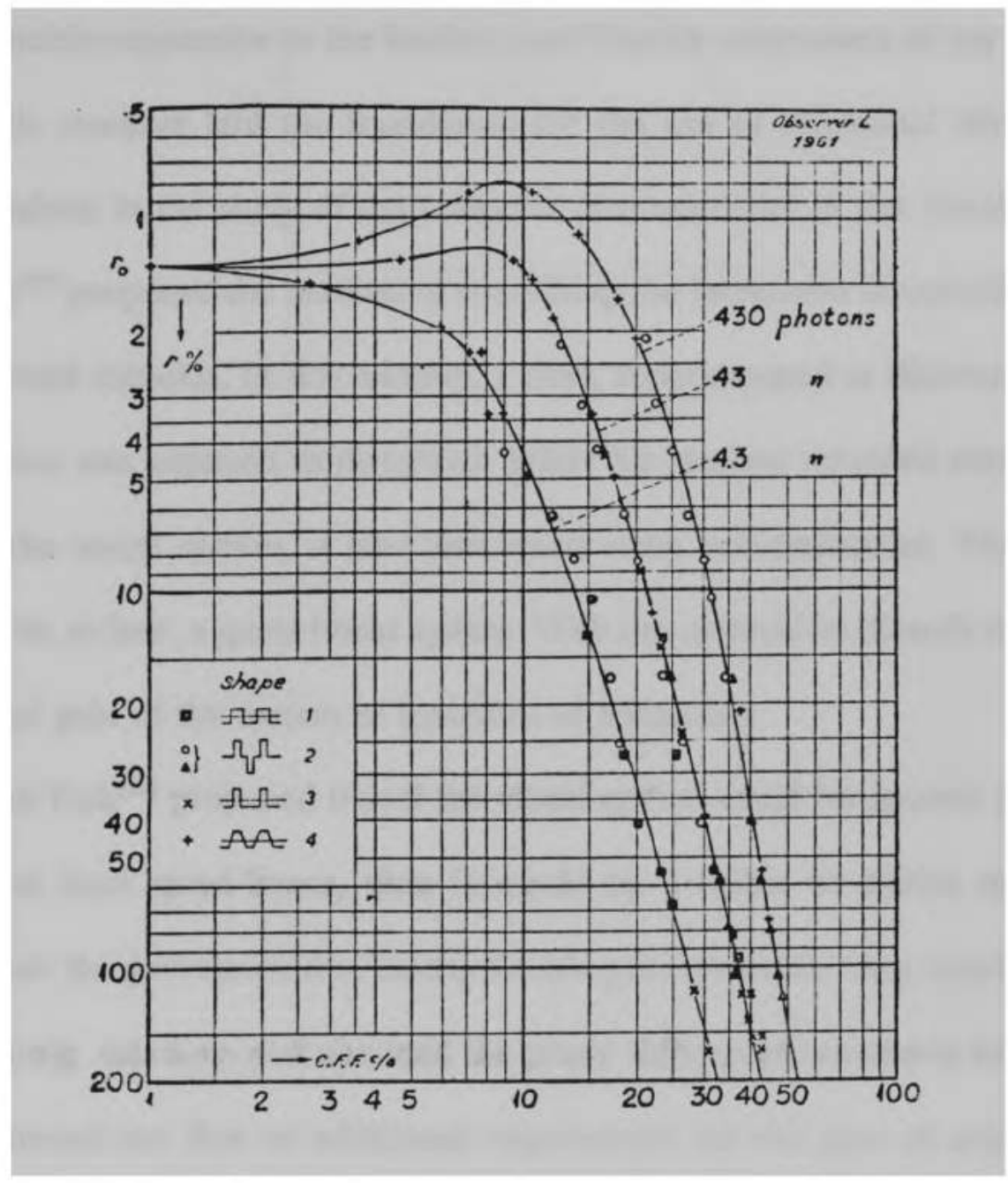

Figure 1.1. Attenuation characteristic of the visual system.

Four types of flicker with the same modulation were applied. (After [6])

Kelly ${ }^{[14]}$ suggested that the visual system behaves like a second-order linear filter, followed by a non-linear stage simulating refractory neural behavior. He successfully 
demonstrated, using the sinusoidal flicker method, that at high frequencies, the visual system was mainly responsive to the fundamental Fourier component of any periodic stimulus. This research laid the foundation for the use of sinusoidal stimuli and harmonic analysis in the study of the temporal characteristics of the visual system. McTavish $^{[15]}$ proposed the method of combining the increment threshold method with a sinusoidal stimulus. In this method, a flash, superimposed at different phases of the stimulus, was adjusted to threshold. While his method revealed many linear features of the visual system, it also uncovered some non-linearities. The system appeared to be, at best, a quasi-linear system. With this method he directly measured the phase and gain of the system as functions of frequency.

Stork and Falk ${ }^{[16]}$ proposed that if the visual system could be treated as stable, causal and at least quasi-linear, then it would be possible to derive the phase spectrum from the gain spectrum. Taking Kelly's gain spectrum, they employed the Kramers-Kronig relations and obtained the phase shift spectrum shown in Fig. 1.2. Victor ${ }^{[17]}$ pointed out that an additional requirement for this type of analysis was that the system must be a minimum-phase system. The curve shows a low frequency phase lead followed by a high frequency phase lag.

Bone ${ }^{[18]}$ et al, using the McTavish method, also obtained gain and phase curves. Their phase spectrum was similar to Stork and Falk's indicating that the system might indeed be a minimum-phase system. Their gain curves were in general agreement with Kelly's measurement. 


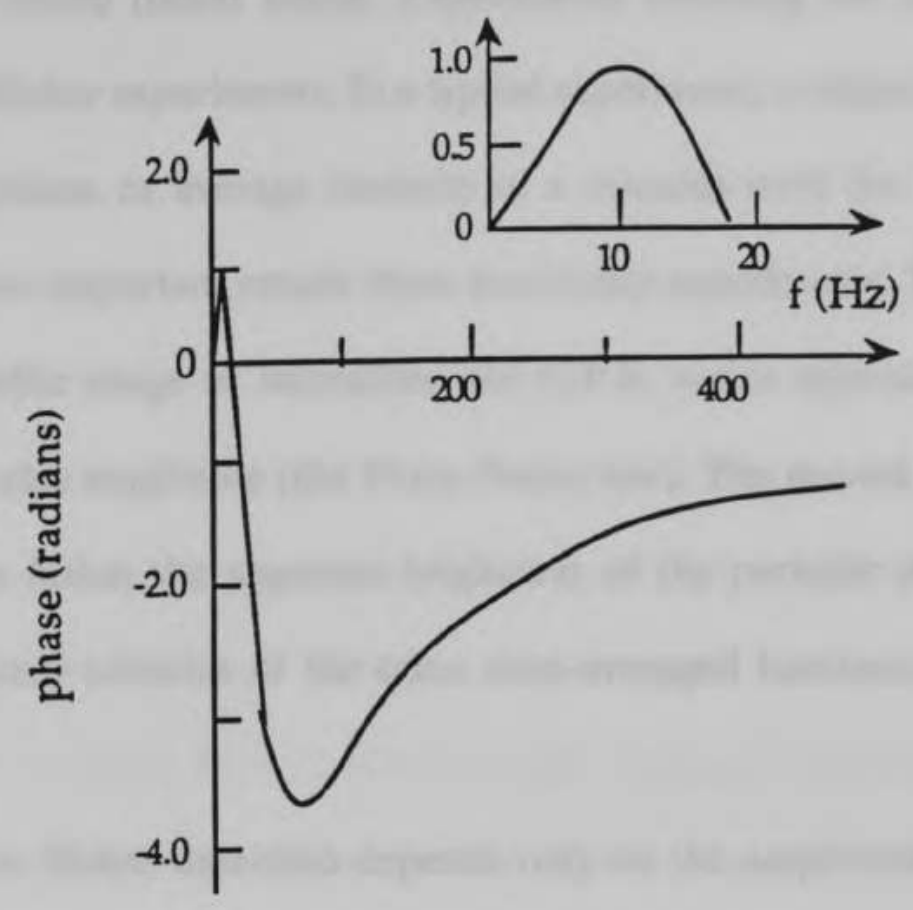

Figure 1.2. Phase shift curve calculation based on

Kelly curve. (After [16])

\subsection{Critical flicker frequency method}

If a visual stimulus flashes repeatedly, it causes the sensation of flicker when its rate is low. This becomes less pronounced as the repetition rate increases. When the conditions are such as to produce the impression of steady illumination, the "fusion point" is said to have been reached. In terms of the flash rate, the "critical flicker frequency" (C.F.F.) has been reached. The phenomenon of flicker disappearance at high 
presentation rates is called flicker fusion. Experiments involving the use of periodic stimuli are known as flicker experiments. In a typical experiment, a subject adjusts either the frequency, modulation, or average intensity of a stimulus until the fusion point is reached. There are two important results from traditional experiments. The first one is that over a considerable range of intensities, the C.F.F. varies approximately as the logarithm of the stimulus amplitude (the Ferry-Porter law). The second one is that for all frequencies above fusion the apparent brightness of the periodic stimulus closely matches that of a steady stimulus of the same time-averaged luminance (the TalbotPlateau law).

If the threshold for flicker detection depends only on the amplitude of the output signal from the system, the gain of the system can be determined as follows. For different frequencies and constant average illuminance, the subject adjusts the modulation until the fusion point is reached. Under these conditions, one can write

Gain, $\mathrm{G}$ = output amplitude/input amplitude

Assuming the output amplitude is constant, regardless of frequency,

$$
\log \mathrm{G}=\text { const. }-\log \mathrm{M} \text {, }
$$

where $\mathrm{M}=$ modulation of input. Thus the curves in Fig. 1.1 can be interpreted as gain spectra. 


\subsection{Increment threshold method}

The increment threshold method is a general method for probing the sensitivity of the visual system under different adaptation conditions. In its simplest application, the stimulus might be a steady, uniformly illuminated visual field of intensity I. A short duration superimposed flash, presented periodically, is then adjusted until a $50 \%$ probability of seeing it is achieved. The flash intensity, $\Delta \mathrm{I}$, is then defined as the "increment threshold" or equivalently its reciprocal the "increment threshold sensitivity."

The experiment yields the type of result illustrated in Fig. 1.3. The two branches reflect responses from the rods and cones respectively. The linear portions of the branches illustrate the Weber-Fechner law:

$$
\Delta \mathrm{I} / \mathrm{I}=\text { constant }
$$

It should be noted that the value of $\Delta \mathrm{I}$ represents a measurement of the output of the visual system under the adaptation conditions determined by the stimulus intensity, I. 


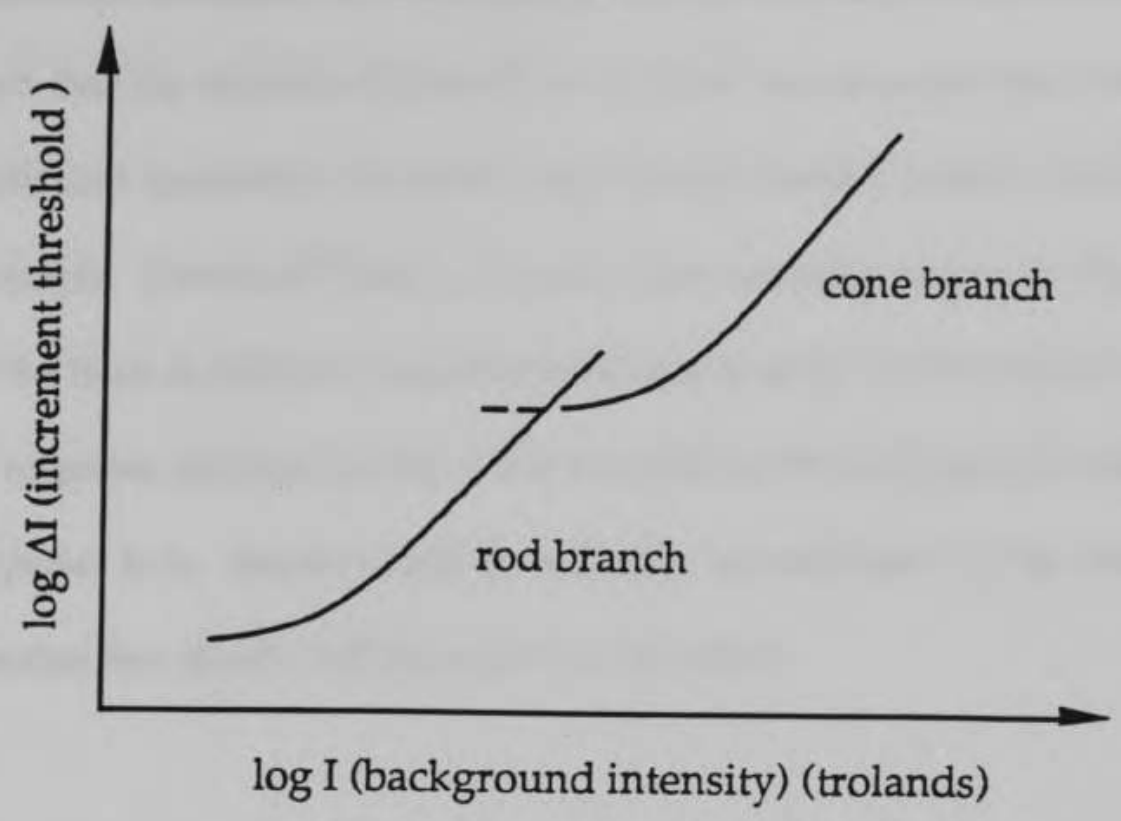

Figure 1.3 The variation of increment threshold with background intensity. (After [19])

The test flash image is usually small and localized in a chosen retinal site such as the fovea. If its duration is short, its temporal shape is not important. Bloch's law states that the time integral of its intensity, i.e. the energy content of the flash, determines its detectability for durations less than $20 \mathrm{msec}$. But, for longer durations, the peak intensity of the flash alone determines detectability.

Thresholds may be grouped into two classes, those which measure the minimal total stimulation required for arousing sensation, and those which measure the minimum detectable difference between objects or two parts of a single object. The former is 
limen" or "differential threshold". It is also called "the just noticeable difference" or j.n.d. Graham showed that the absolute threshold is no lower for two eyes than for one ${ }^{[20]}$.

More complicated increment threshold experiments involve stimuli which are not steady. For example, Crawford ${ }^{[21]}$ used a square pulse stimulus shown in Fig. 1.4 and superimposed the flash at different temporal positions in order to determine the system response. The response sketched in Fig. 1.4 is elevated at the leading and trailing edges of the square pulse. It is characterized by a strong "on-response" to the onset of the pulse and a weaker but slower "off-response" to the offset.

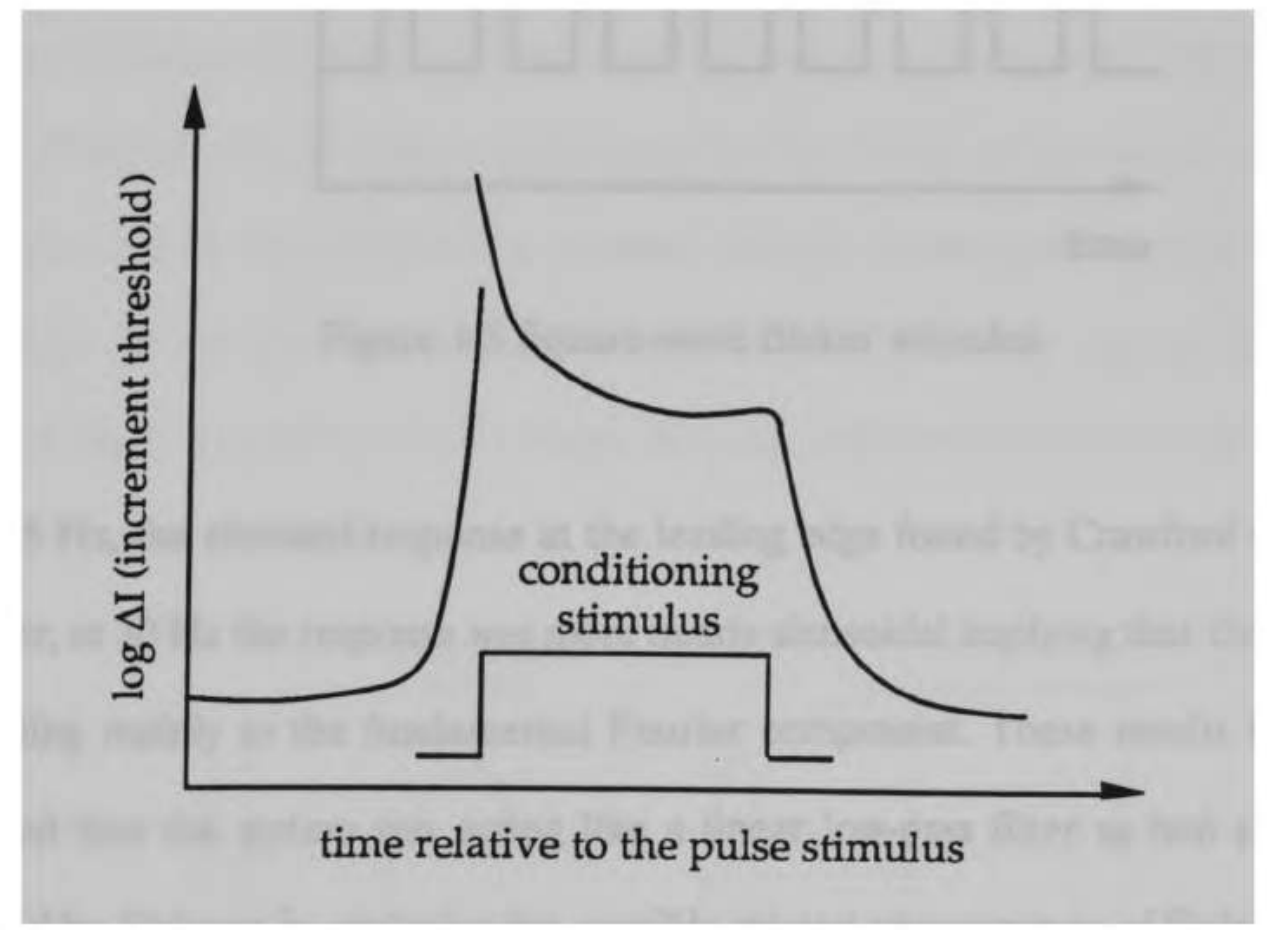

Figure 1.4 Foveal thresholds measured before, during and after the onset of pulse. (After[21]) 


\section{Combined increment threshold and flicker method}

An extension of Crawford's work, using a continuous square wave stimulus shown in Fig. 1.5, was carried out by Boynton, Sturr and Ikeda ${ }^{[22]}$. In their experiment, a test flash was no longer presented with each pulse but instead only once per second in order that the possible flicker fusion of the test flash would not interfere with the threshold measurement.

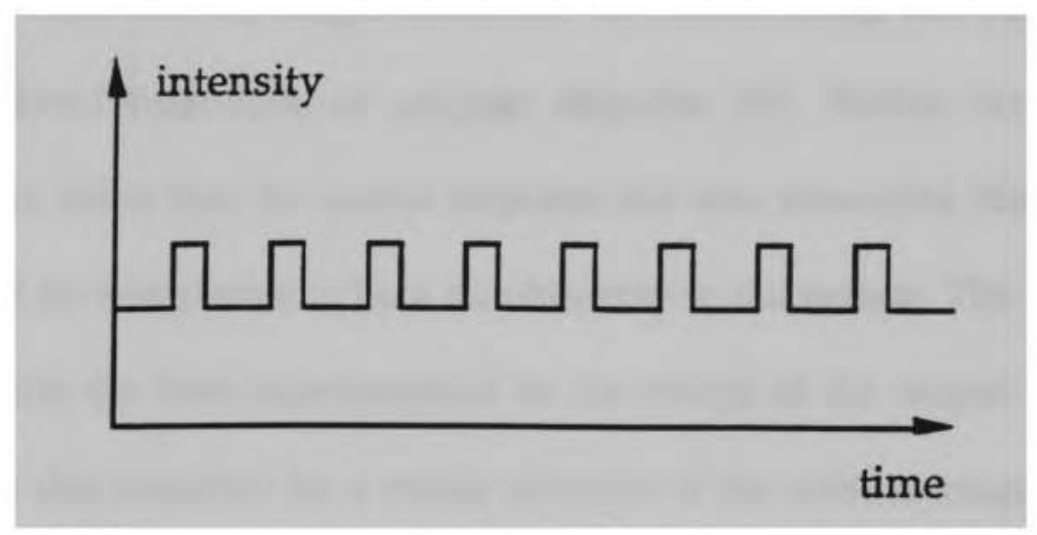

Figure 1.5 Square-wave flicker stimulus

At $15 \mathrm{~Hz}$, the elevated response at the leading edge found by Crawford was evident. However, at $30 \mathrm{~Hz}$ the response was more nearly sinusoidal implying that the system was responding mainly to the fundamental Fourier component. These results immediately suggested that the system was acting like a linear low-pass filter as had already been proposed by Delange in analyzing the possibly related phenomenon of flicker fusion. By reducing the intensity of the flicker stimulus and measuring the test flash threshold when it was superimposed at the midpoint of the dark and of the bright phases of the waveform, Boynton ${ }^{[23]}$ found two important results: 
1. The existence of a phase shift between the visual responses to the test flash and the stimulus at that point in the visual system at which the threshold is determined.

2. The test flash threshold detection is performed prior to flicker detection in a sequential processing system.

McTavish ${ }^{[15]}$ was the first person to design an experimental system using sinusoidal stimuli with the increment threshold method. In this system, the test flash was set at the center of the field of view with an image size of $1.7^{\circ}$ as shown in Fig. 1.6. The stimulus was a spatially uniform visual field of angular diameter $18^{\circ}$. Within the limits of experimental error, he found that the system response was also sinusoidal. However, his work uncovered what he interpreted to be a non-linearity in the system. The increment threshold obtained with the flash superimposed on the trough of the output was found to be elevated above that required for a steady stimulus of the same average intensity. The variation of the peak and trough thresholds with frequency that he found are sketched in Fig. 1.7(a). For a linear system, the peak and trough curves would have the symmetry shown in Fig 1.7(b).

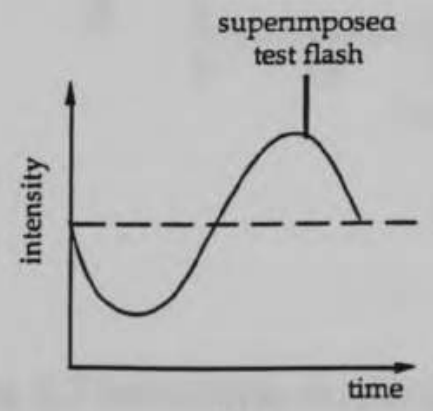

(a)

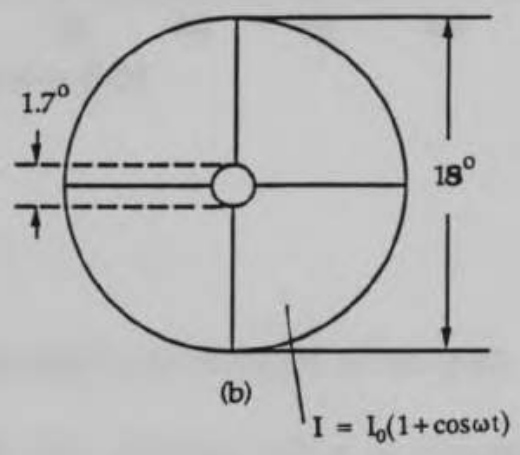

Figure 1.6 Visual field 


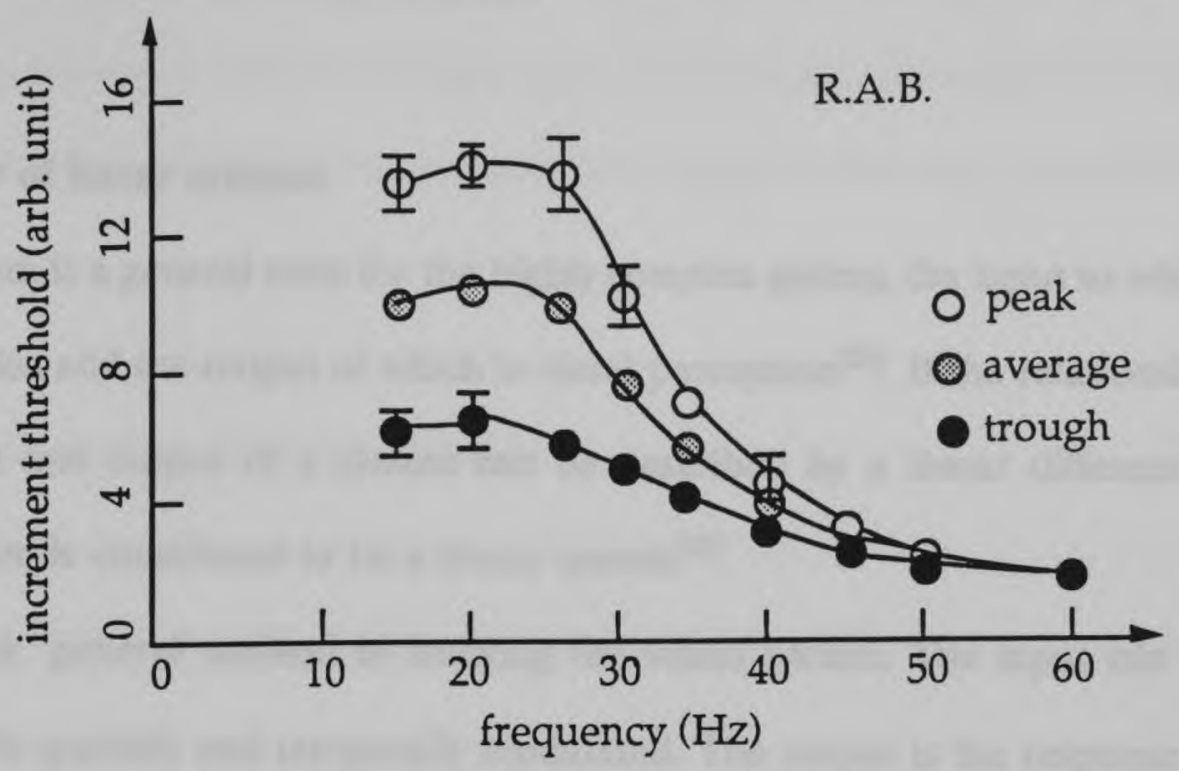

(a)

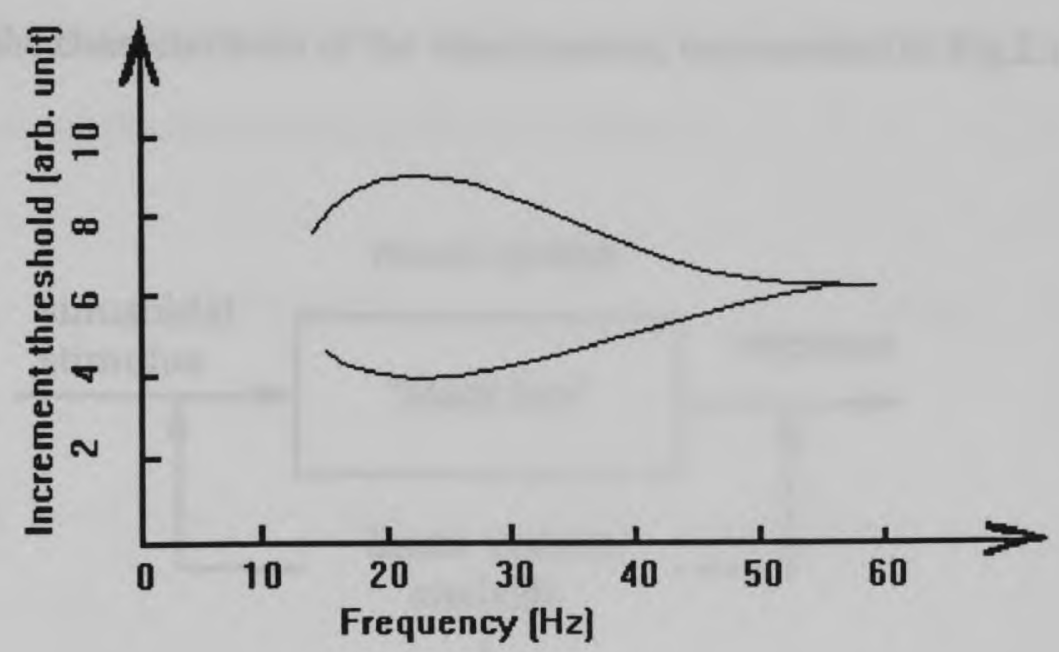

(b)

Figure 1.7 Increment threshold at peak and trough of output.

(a) Experimental result: the average value is elevated.

(b) Theoretical result assuming linearity. 


\section{Chapter 2. Theoretical Considerations}

\subsection{General theory of linear systems}

The visual system is a general term for the highly complex system, the input to which is an optical stimulus and the output of which is visual perception ${ }^{[24]}$. If the relationship between the input and output of a system can be described by a linear differential equation, the system is considered to be a linear system ${ }^{[25]}$.

Fig. 2.1 shows a general method of studying the visual system. The input can be various stimuli both spatially and temporally modulated. The output is the response of the visual system which can be experimentally determined. Investigation of the output response may reveal the characteristics of the visual system, represented in Fig.2.1 by the black box.

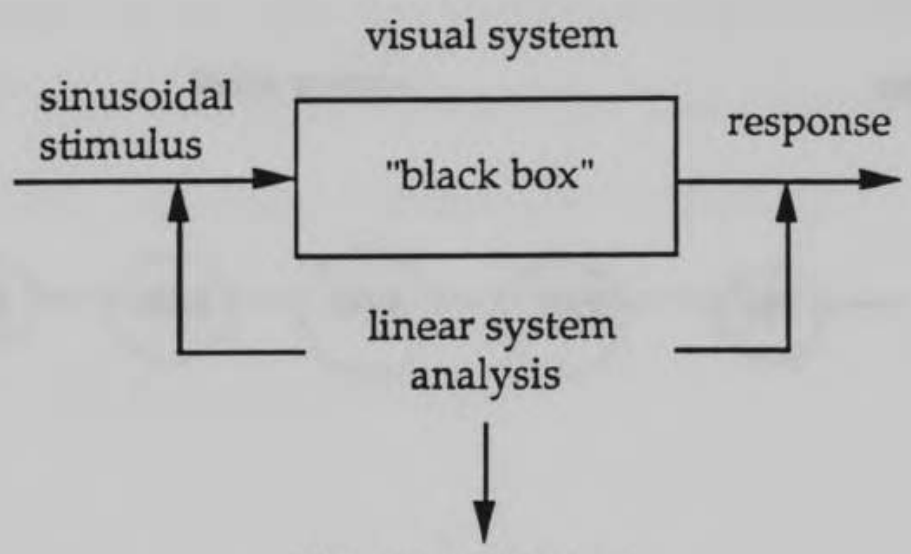

vision mechanism

Figure 2.1. "Black box" model for the visual system 
Generally, a linear system is also defined as one which obeys the principle of superposition: If an input $x_{1}(t)$ causes an output $y_{1}(t)$, and an input $x_{2}(t)$ causes an output $\mathrm{y}_{2}(\mathrm{t})$, then an input $\mathrm{x}_{1}(\mathrm{t})+\mathrm{x}_{2}(\mathrm{t})$ will give an output $\mathrm{y}_{1}(\mathrm{t})+\mathrm{y}_{2}(\mathrm{t})$. In operator terminology, that means:

$$
\mathscr{F}\left[\mathrm{x}_{1}(\mathrm{t})+\mathrm{x}_{2}(\mathrm{t})\right]=\mathscr{F}\left[\mathrm{x}_{1}(\mathrm{t})\right]+\mathscr{F}\left[\mathrm{x}_{2}(\mathrm{t})\right]
$$

If this equation is satisfied for all functions $\mathrm{x}_{1}(\mathrm{t})$ and $\mathrm{x}_{2}(\mathrm{t})$, then $\mathscr{F}$ is said to be a linear operator.

When the visual system behaves linearly, according to linear systems analysis the response to any arbitrary stimulus can be calculated by using Fourier analysis. Fig. 2.2 shows a block diagram of the treatment by Fourier analysis.

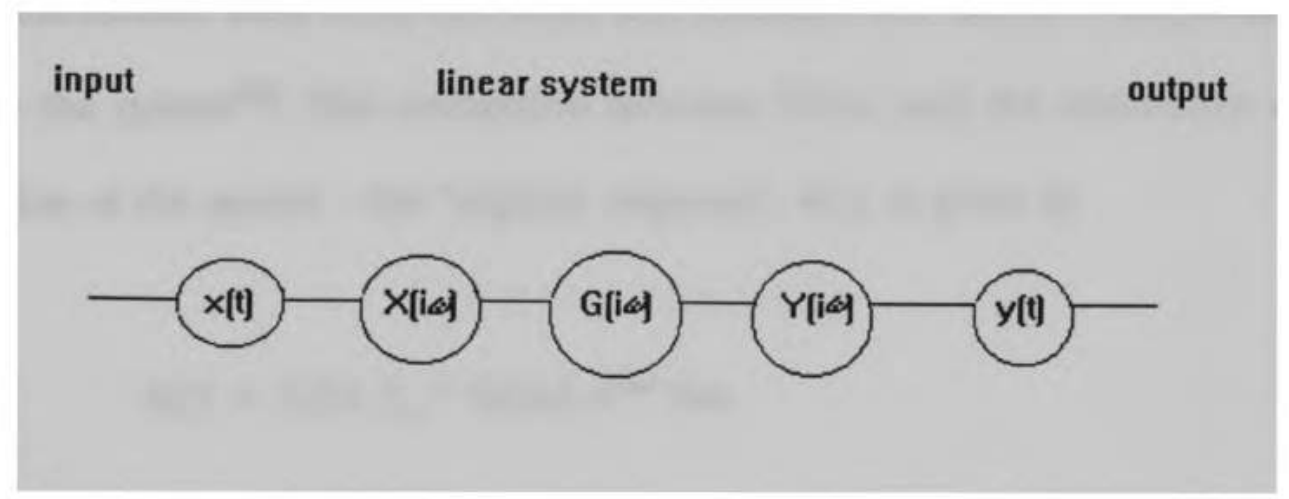

Figure 2.2. Fourier analysis for a linear system 
If the input of a system is $x(t)$ and its complex Fourier transform is $X(i \omega)$, and if $y(t)$ is the output function and $\mathrm{Y}(\mathrm{i} \omega)$ is its complex Fourier transform, then the "frequency transfer function" $\mathrm{G}(\mathrm{i} \omega)$, of the linear system is given by

$$
\mathrm{G}(\mathrm{i} \omega)=\mathrm{Y}(\mathrm{i} \omega) / \mathrm{X}(\mathrm{i} \omega)
$$

$\mathrm{G}(\mathrm{i} \omega)$ reflects the frequency response characteristic of the linear system and provides a complete description of the system. Such a complex function can also be described by

$$
\mathrm{G}(\mathrm{i} \omega)=\mathrm{A}(\omega) \exp [\mathrm{i} \Phi(\omega)]
$$

$A(\omega)=|G(i \omega)|$ represents the gain characteristic of the system and $\Phi(\omega)$ is the phase shift characteristic. Both $\mathrm{A}(\omega)$ and $\Phi(\omega)$ are functions of $\omega$ but are independent of the input to the system ${ }^{[26]}$. The connection between $\mathrm{G}(\mathrm{i} \omega)$ and the alternative complete description of the system - the "impulse response", $\mathrm{h}(\mathrm{t})$, is given by

$$
h(t)=1 / 2 \pi \int_{-\infty}^{\infty} G(i \omega) e^{-i \omega t} d \omega,
$$

i.e. $h(t)$ is the inverse Fourier transform of $G(i \omega)$.

Two simple examples serve to illustrate some important results.

1. If the system consists of a first order derivative element, the transfer function is 


$$
\mathrm{G}(\mathrm{i} \omega)=\mathrm{i} \omega+\mathrm{a}
$$

with the corresponding gain function being

$$
A(\omega)=\left(\omega^{2}+a^{2}\right)^{1 / 2}
$$

and the phase shift being

$$
\Phi(\omega)=\tan ^{-1}(\omega / \mathrm{a})
$$

The derivative element represents a high-pass filter for sinusoidal inputs. Relative to the input, the output has a phase lead approaching a constant value of $\pi / 2$ at high frequency.

2. For a first order integrative element,

$$
G(i \omega)=1 /(i \omega+b) .
$$

The gain is

$$
\mathrm{A}(\omega)=1 /\left(\omega^{2}+\mathrm{b}^{2}\right)^{1 / 2}
$$

and the phase shift is 


$$
\Phi(\omega)=\tan ^{-1}(-\omega / b)
$$

The integrator is a low-pass filter with a phase lag approaching $\pi / 2$ at high frequency. Any linear system consists of derivative elements and/or integrative elements in series, parallel, or feedback combinations. Generally, the frequency transfer function can be denoted by

$$
\mathrm{G}(i \omega)=\left\{\left(i \omega+a_{1}\right) \ldots\left(i \omega+a_{m}\right)\right\} /\left\{\left(i \omega+b_{1}\right) \ldots\left(i \omega+b_{n}\right)\right\}
$$

The numerator shows the order of the derivative elements in the system and the denominator shows the order of the integrative elements in the system. Using Bodeplots, some characteristics of the system may be approximated as follows:

At low frequency, the frequency transfer function

$$
\mathrm{G}(\mathrm{i} \omega) \approx \text { constant }
$$

This means that the gain of the system is constant and produces a zero phase shift. At high frequency,

$$
\mathrm{G}(\mathrm{i} \omega) \approx 1 /(\mathrm{i} \omega)^{(\mathrm{m}-\mathrm{n})} \exp [\mathrm{i}(\mathrm{m}-\mathrm{n})(\pi / 2)]
$$

From this, 


$$
A(\omega)=\{|G(i \omega)|\}=1 /(\omega)^{(m-n)} .
$$

or

$$
\log A(\omega)=-(m-n) \log (\omega)
$$

and

$$
\Phi(\omega)=(m-n)(\pi / 2)
$$

The slope of the gain curve at high frequency on a Bode-plot therefore gives the order (m-n) of the linear system. Alternatively, the order is obtained from the high frequency phase shift.

Of course not all systems are completely linear. In many, the phenomenon of saturation occurs where the magnitude of the response is more or less abruptly restricted to a limiting value. If a system has been constructed with the intention of its being a linear system, the departure from linearity with increasing input level is called non-linear distortion. Useful information about such a system can still be obtained by ensuring that inputs to the system do not evoke such non-linear behavior. 


\subsection{Diffusion model}

A model of considerable interest which has been proposed to account for a number of experimental results in temporal studies is the diffusion model. Flicker data, including in particular Delange's ${ }^{[27]}$, revealed gain functions with ever increasing high frequency slopes. Such behavior is not obtained from a system of cascaded filters which gives rise to a high frequency asymptote and a transfer function in the form of a power function. On the other hand it is consistent with a diffusion system which has an exponential transfer function.

The diffusion model is attractive because it is easy to understand how it could represent physiological processes, for example the movement of photo-products within a receptor cell. Kelly ${ }^{[28]}$ showed the model gave a much better fit to the flicker data than either the Fuortes-Hodgkin ${ }^{[29]}$ model (cascaded integrator with feed-back control of the gain and time constants) and the Ferry-Porter law (see 1.2). Ives ${ }^{[2]}$ first suggested the idea of a linear diffusion process either governing the transport of photo-products, or a membrane ionic current. Veringa ${ }^{[30]}$ obtained phase data which supported the model by using electrically induced "phosphenes" (visual responses not produced by light). While simultaneously applying to the retina sinusoidal light and sinusoidal electrical stimuli, the relative phase between them was adjusted to produce no sensation of flicker. Assuming the response to the electrical stimulus was essentially instantaneous, the phase shift for the light signal could be determined. This was found to have the form of a phase lag proportional to the square root of the frequency, completely in accord with the IvesVeringa theory as shown below. 
The diffusion model first considers, for example, the transfer of molecules by a diffusion process. This may be described by the diffusion equation

$$
\partial \mathrm{f} / \partial \mathrm{t}=\mathrm{k} \partial^{2} \mathrm{f} / \partial \mathrm{x}^{2}-\rho \mathrm{f}
$$

where $f=f(x, t)$ is the concentration within a one dimensional diffusion cell $(0<x<\infty)$, and the flux in the cell is proportional to $\partial \mathrm{f} / \partial \mathrm{x}$. The constant $\mathrm{k}$ is associated with the rate of diffusion and $\rho$ with the rate of concentration loss or leakiness of the cell. By applying reasonable boundary conditions, the transfer function, $G(i \omega)$, can be obtained through the use of the Laplace transform:

$$
\begin{aligned}
G(i \omega) & =\exp \left[-(\omega \tau)^{1 / 2}(1 \pm i)\right] \\
& =\exp \left[-(2 i \omega \tau)^{1 / 2}\right]
\end{aligned}
$$

where $\tau$ is a time constant. $(\mp \omega)^{1 / 2}$ can always be taken as a real number, because $(\mp 2 \mathrm{i})^{1 / 2} \equiv 1 \mp \mathrm{i}$.

This gives a gain,

$$
|\mathrm{G}(\mathrm{i} \omega)|=\exp \left[-(\omega \tau)^{1 / 2}\right]
$$

and a phase lag, 


$$
\Phi(\omega)=-(\omega \tau)^{1 / 2}
$$

As will be shown later, this parabolic phase function is not in accord with the results of the present study.

Considering both diffusion and inhibition processes, Kelly ${ }^{[31]}$ proposed a more complex theory which accounted for the flicker data at all frequencies over a wide range of photopic adaptation levels. This model had the following properties:

(1) Its high-frequency behavior was controlled by a linear process, whereas its lowfrequency behavior was controlled by non-linear neural processes.

(2) A single transfer function with variable parameters represented the entire model.

(3) It predicted reasonable impulse responses with sensitivities and time constants that varied appropriately with adaption level.

The model involved two stages: one was a diffusion stage and the other represented inhibition processes (non-linear feedback stages). It is shown in Fig.2.3.

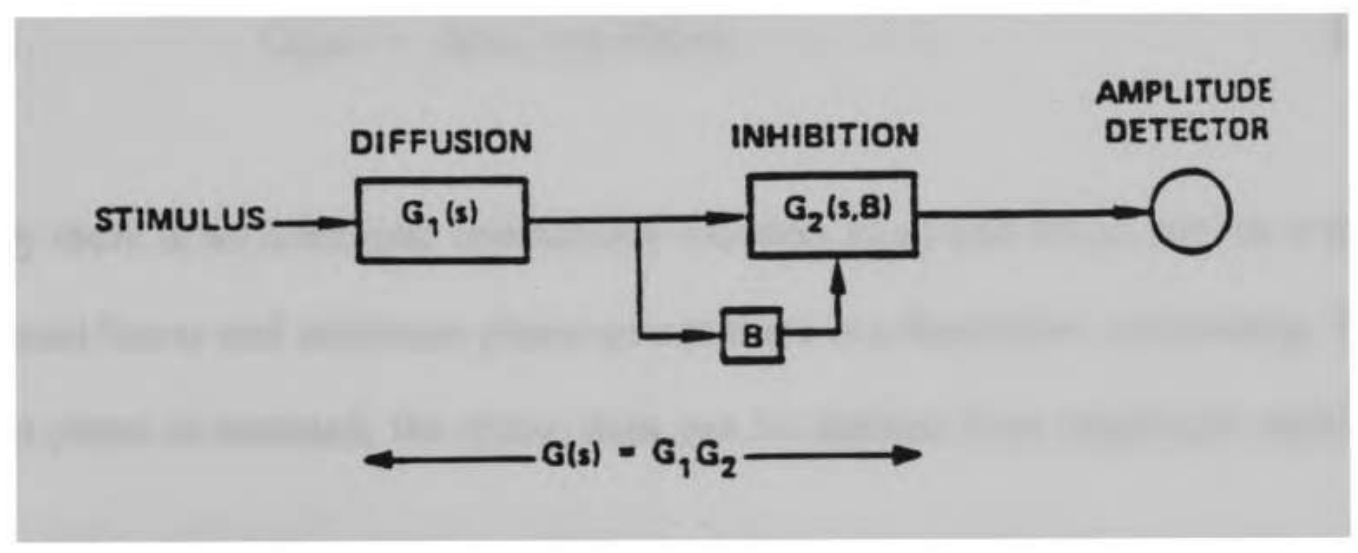

Figure 2.3. Schematic diagram of the proposed two-stage model.

However, the expression for the phase shift still had the form of equation (2.7) 


\subsection{The relationship between gain and phase}

A concept of considerable importance to this study is that of a "minimum phase" system. In fact it was thought that the visual system might fall into this category. A minimum phase system is characterized as one having neither poles nor zeros in the right half s-plane $e^{[32]}$. The name derives from the fact that a stable system having a zero at $\mathrm{s}=\mathrm{a}$ may be converted to one having the same gain but reduced phase lag by multiplying the system function by a factor $(s+a) /(s-a)$.

There is an interesting qualitative difference between a minimum phase transfer function and a corresponding non-minimum phase transfer function. The impulse response that corresponds to the former tends to have fewer zero crossings than the impulse response to the latter. The plausibility of the visual system possibly being a minimum phase system may therefore be examined in the light of impulse response data.

Consider the transfer function in its polar form:

$$
\mathrm{G}(\mathrm{i} \omega)=\mathrm{A}(\omega) \exp \mathrm{i} \Phi(\omega)
$$

Generally there is no functional relationship between $A(\omega)$ and $\Phi(\omega)$, but for a stable, causal, quasi-linear and minimum phase system there is a functional relationship. When minimum phase is assumed, the phase data can be derived from amplitude data ${ }^{[33]}$ as follows:

Suppose a new quantity is defined as 


$$
\mathrm{a}(\omega)=\ln [\mathrm{A}(\omega)]
$$

called the logarithmic gain. Then from (2.8)

$$
\ln [\mathrm{G}(\mathrm{i} \omega)]=\mathrm{a}(\omega)+\mathrm{i} \Phi(\omega)
$$

i.e. $\mathrm{a}(\omega)$ and $\Phi(\omega)$ are the real and imaginary parts of $\ln [\mathrm{G}(\mathrm{i} \omega)]$. The relation between them for a minimum phase system can be established by a contour integration. In this operation, the special feature of the minimum phase function is that not only $G(i \omega)$, but also $\ln [\mathrm{G}(\mathrm{i} \omega)]$ is analytic at all points in the right-half s-plane ${ }^{[34]}$.

Although apparently unaware of this feature, Stork and Falk ${ }^{[16]}$ employed the Kramers-Kronig relations to derive a phase spectrum from Kelly's amplitude data. (Victor ${ }^{[17]}$ later pointed out that their derivation required that the system be a minimum phase system, as well as a stable, causal one.) In order to be able to use numerical integration techniques, they modified equation (2.9) to read

$$
\mathrm{E}(\omega) \equiv \ln [\mathrm{G}(\mathrm{i} \omega)]=\mathrm{a}(\omega)+\mathrm{i} \Phi(\omega)-\mathrm{F} .
$$

F was a subtraction constant to ensure that $\mathrm{E}(\omega)$ vanished at the limits of integration, $\pm \omega_{\max }$, chosen arbitrarily to be $\pm 500 \mathrm{~Hz}$. They also artificially extended Kelly's amplitude data beyond the $\sim 0-50 \mathrm{~Hz}$ range with a function of the form $\mathrm{B}\left(\omega^{2}+\mathrm{b}^{2}\right)^{-1 / 2}$. $\mathrm{B}$ and $\mathrm{b}$ were chosen to ensure continuity of the resulting function and its first derivative 
with the experimental data.

Thus

$$
F=\ln \left[B\left(\omega_{\max }{ }^{2}+b^{2}\right)^{-1 / 2}\right]
$$

$\mathrm{E}(\boldsymbol{\omega})$ is analytic in the right-half s-plane, and its real part is known from the measured amplitude spectrum. According to the Kramers-Kronig relation

$$
\begin{aligned}
\Phi(\omega)= & \operatorname{Im}[\mathrm{E}(\omega)]=(-1 / \pi) f_{-\infty}^{\infty}\left(\operatorname{Re}\left[\mathrm{E}\left(\omega^{\prime}\right)\right] / \omega-\omega^{\prime}\right) \mathrm{d} \omega^{\prime} \\
& =(-1 / \pi) f_{-\infty}^{\infty}\left(\ln \left|\mathrm{G}\left(\omega^{\prime}\right)\right|-\mathrm{F}\right) /\left(\omega^{\prime}-\omega^{\prime}\right) \mathrm{d} \omega^{\prime}
\end{aligned}
$$

(The double slash through the integral sign refers to the Cauchy Principal Value.) This equation describes the relationship between the phase shift and gain of a linearly analytical system with minimum phase. The phase shift and the gain of such a system are both independent of any physiological model and contain no free parameters. When the equation was applied to the gain curve of Kelly ${ }^{[14]}$, the phase curve reproduced in Fig.

\section{2 was obtained.}

Noting the similarity between this curve and preliminary phase measurements obtained by increment thresholds, Tang ${ }^{[35]}$ suggested that the visual system might be a minimum phase system. However, his uncertainties were too large at high frequencies to establish this unambiguously. 


\section{Chapter 3. Experiments}

\subsection{Experimental requirements}

From previous studies ${ }^{[36-40]}$, it is known that the initial stages of the visual system which produce the threshold response act in a quasi-linear manner at relatively high frequency. It is therefore reasonable to apply the standard techniques of linear analysis using this output response of the system. This involves applying a sinusoidal input to the system and observing the gain and phase shift of the resulting sinusoidal output. Nonlinearities might show up in a number of ways, in a particular, a distortion of the output waveform from that of a true sinusoid. It was therefore desirable to determine the entire output waveform using the increment threshold method.

The experiment was designed with the following considerations in mind:

1. Input to the visual system should be provided by an achromatic stimulus with a wide, spatially uniform field. The stimulus intensity should be sinusoidally modulated in time over the frequency range $0-60 \mathrm{~Hz}, 60 \mathrm{~Hz}$ being above the fusion point for the intensities used. The percentage modulation should be variable in the $0-100 \%$ range, but with the average intensity held constant in the photopic range. This ensures that the adaptation state remains constant, responses being cone-mediated.

2. In the increment threshold technique, the visibility of a superimposed test flash on the stimulus provides a measure of the sensitivity of the system or, alternatively, the output. The test flash should be small and centrally located so that the response is 
essentially a cone response only. Its duration should be very short compared with the period of the highest stimulus frequency: i.e. $<<20 \mathrm{~ms}$. It should be presented at a sufficiently low repetition rate to ensure that each flash is processed independently. The intensity of the flash should be easily adjustable by the subject. Finally, the temporal position of the flash with respect to the stimulus cycle should be continuously adjustable. 


\subsection{Experimental system}

In order to satisfy the previously stated requirements, an experimental system containing three main parts was designed. These were:

1. a sinusoidal visual signal generator

2. a test flash system

3. a control system.

Fig. 3.1 is a diagram of the experimental system

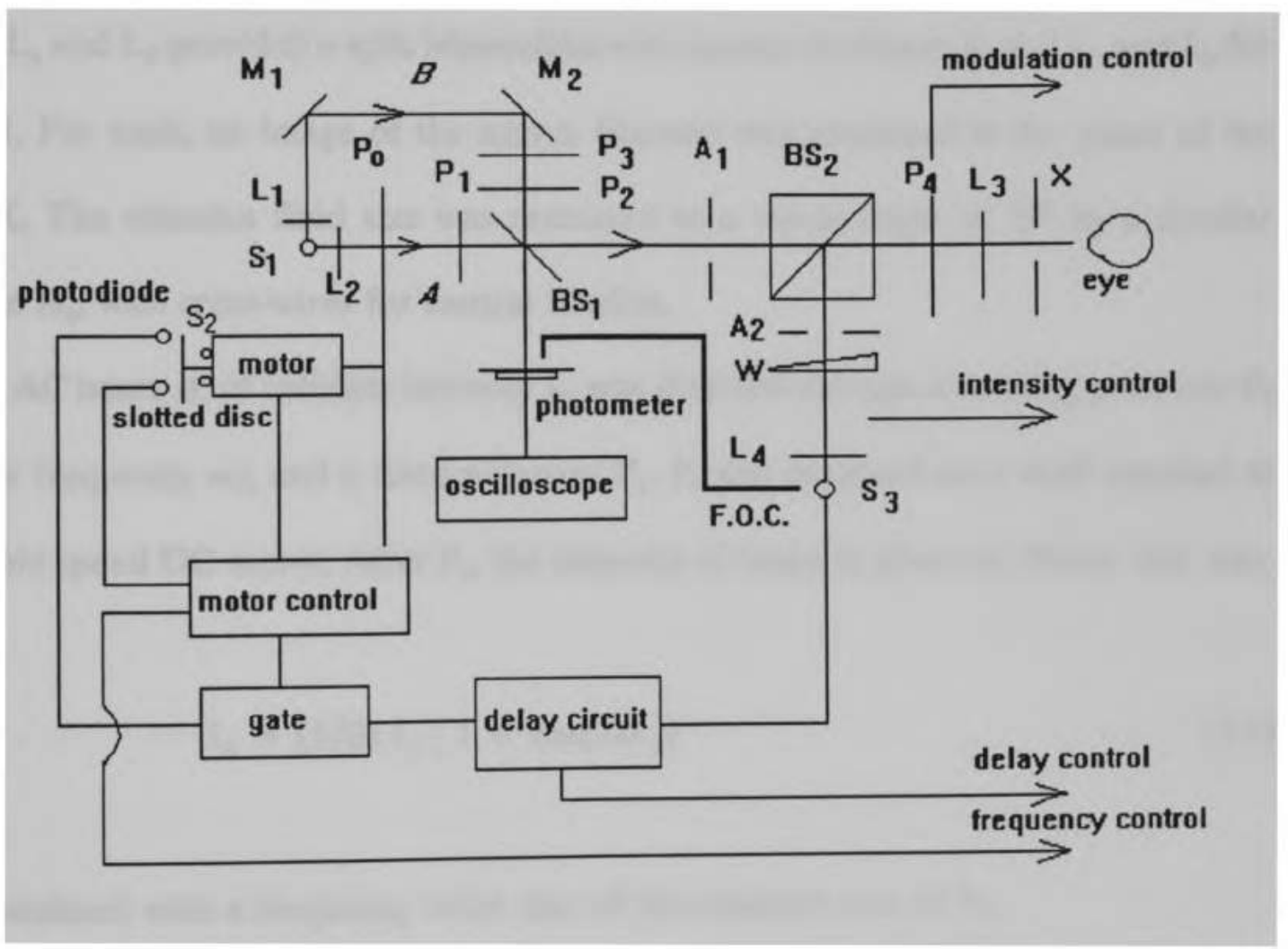

Figure 3.1 Experiment system 


\section{The visual signal generator}

The visual signal generator ${ }^{[31]}$ provided a stimulus of independently controllable frequency, amplitude and intensity. The optical system employed Maxwellian view since this yields a retinal illuminance as high as that for direct viewing of the light source, but with a larger area of the retina being illuminated ${ }^{[41]}$. Two light beams, $A$ and $B$, were derived from a quartz-halogen light source $\mathrm{S}_{1}$ and recombined by beam splitter $\mathrm{BS}_{1} . A$ and $B$ provided the AC and DC components respectively of the sinusoidal stimulus. Lenses $\mathrm{L}_{1}$ and $\mathrm{L}_{3}$ provided a split Maxwellian view system for beam $B$, and $\mathrm{L}_{2}$ and $\mathrm{L}_{3}$ for beam $A$. For each, an image of the source filament was produced in the plane of the pupil, X. The stimulus field size was restricted to a visual angle of $15^{\circ}$ by a circular aperture $A_{1}$, with cross-wires for central fixation.

The AC beam $A$, of incident intensity $\mathrm{I}_{1}$, was directed through a rotating polarizer $\mathrm{P}_{0}$ (angular frequency $\omega$ ), and a fixed polarizer $P_{1} . P_{0}$ was mounted on a shaft coupled to a variable speed DC motor. After $\mathrm{P}_{1}$, the intensity of beam $A$, given by Malus' law, was

$$
I_{A}=(1 / 2) I_{1}[1+\cos (2 \omega t)]
$$

i.e., modulated with a frequency twice that of the rotation rate of $\mathrm{P}_{0}$.

The DC beam $B$, of incident intensity $\mathrm{I}_{2}$, passed through two fixed polarizers, $\mathrm{P}_{3}$ and $P_{2}$. If the angle between the polarization directions of $P_{3}$ and $P_{2}$ was $\phi$, then the intensity of the DC beam, also given by Malus' law, was: 


$$
\mathrm{I}_{\mathrm{B}}=\mathrm{I}_{2} \cos ^{2} \phi
$$

The polarization directions of $\mathrm{P}_{1}$ and $\mathrm{P}_{2}$ were adjusted to be perpendicular to each other. Another polarizer, $\mathrm{P}_{4}$, was used to adjust the modulation of the stimulus. If the angle between $P_{1}$ and $P_{4}$ was $\theta_{1}$, then the angle between $P_{2}$ and $P_{4}$ was $\theta_{2}=\left(90-\theta_{1}\right)$. Therefore after $\mathrm{P}_{4}$, the intensities of beams $A$ and $B$ were

$$
I_{A}=(1 / 2) I_{1}[1+\cos (2 \omega t)] \cos ^{2}\left(\theta_{1}\right)
$$

and

$$
I_{B}=I_{2} \cos ^{2}(\phi) \cos ^{2}\left(\theta_{2}\right)=I_{2} \cos ^{2}(\phi) \sin ^{2}\left(\theta_{1}\right)
$$

The intensity of the sinusoidal stimulus at the final pupil, $\mathrm{X}$, was:

$$
\begin{aligned}
I= & I_{A}+I_{B} \\
= & {\left[I_{2} \cos ^{2}(\phi) \sin ^{2}\left(\theta_{1}\right)+(1 / 2) I_{1} \cos ^{2}\left(\theta_{2}\right)\right.} \\
& \left.+(1 / 2) I_{1} \cos ^{2}\left(\theta_{1}\right) \cos (2 \omega t)\right]
\end{aligned}
$$

In order that the modulation of the stimulus could be changed while the average 
intensity remained constant, the angle $\phi$ was set to meet the condition:

$$
\begin{aligned}
\mathrm{I}_{2} \cos ^{2}(\phi) & =(1 / 2) \mathrm{I}_{1} \\
& =\mathrm{I}_{0}
\end{aligned}
$$

i.e. the average intensity of the AC beam was the same as the (constant) intensity of the DC beam. Then equation (3.5) becomes:

$$
\begin{aligned}
I & =\left[I_{0} \sin ^{2}\left(\theta_{1}\right)+I_{0} \cos ^{2}\left(\theta_{1}\right)\right]+I_{0} \cos ^{2}\left(\theta_{1}\right) \cos (2 \omega t) \\
& =I_{0}\left[1+\cos ^{2}\left(\theta_{1}\right) \cos (2 \omega t)\right]
\end{aligned}
$$

Thus the signal generator produced a sinusoidal stimulus with an average intensity $I_{0}$, variable amplitude $I_{0} \cos ^{2}\left(\theta_{1}\right)$, and frequency $2 \omega$.

Adjustable forehead and chin rests were provided in order to keep the subject's right eye fixed with respect to the artificial pupil X. This was $1.5 \mathrm{~mm}$ diameter, smaller than the eye's natural pupil, and therefore controlled the retinal illuminance.

\section{Test flash system}

The test flash was provided by a xenon flash tube $\mathrm{S}_{3}$. The duration of the flash was $0.94 \mathrm{~ms}$, thereby fulfilling the requirement noted earlier. Lenses $\mathrm{L}_{4}$ and $\mathrm{L}_{3}$ provided a split Maxwellian view system. $\mathrm{BS}_{2}$ represented the stacked beam-splitters shown in Fig. 3.2 and was used to introduce the flash. This design eliminated any polarization of the 
flash which a single beam-splitter would produce, and which would be affected by rotation of $\mathbf{P}_{4}$. The size of the centrally viewed test flash field was limited to a visual angle of $1.7^{\circ}$ by a small circular aperture, $A_{2}$. The flash intensity was controlled by neutral wedge, W. W was moved by a stepper-motor under the control of the subject. Forward and reverse push-buttons on a hand-held control adjusted the optical density of the wedge in 0.01 increments. A third button allowed a reading to be recorded.

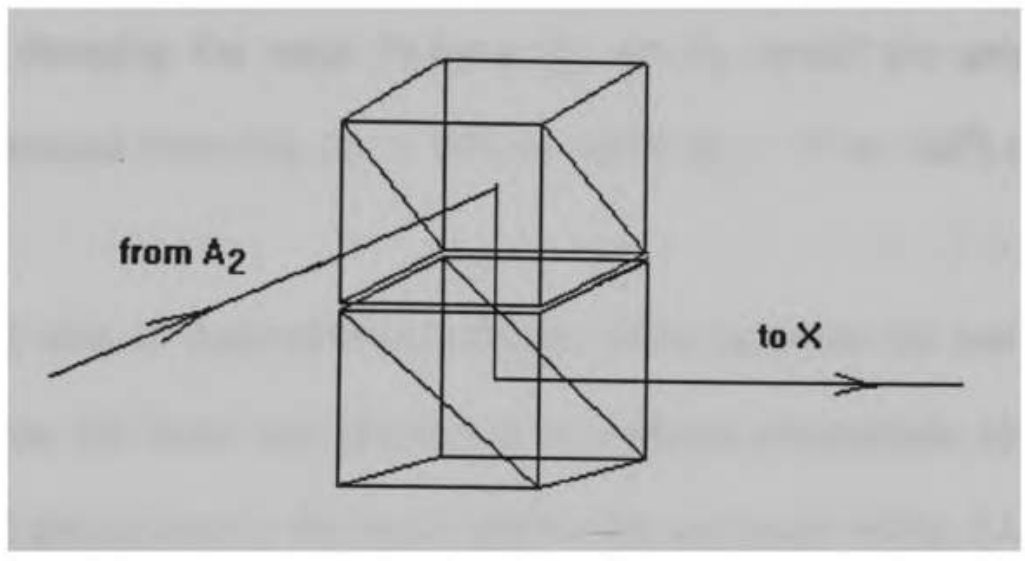

Figure 3.2 Diagram of stacked beam-splitters

The xenon flash tube $S_{3}$ was to be triggered at precisely controlled instants relative to the sinusoidal stimulus. To achieve this, a disc containing a single radial slot was attached to the motor shaft for $\mathrm{P}_{0}$ between a small light source $\mathrm{S}_{2}$ and a photodiode. The photodiode output was therefore a series of pulses of which approximately one per second was allowed to pass through an electronic gate to an adjustable delay circuit. The output pulse from the delay circuit was then used to trigger the flash lamp $\mathrm{S}_{3}$. Adjustment of a potentiometer, either by the subject or by the operator, allowed the flash to be located at any temporal position in the stimulus cycle. 


\section{Control System}

The motor speed was measured by a second slotted disc-light source-photodiode arrangement which provided 12 pulses per revolution to a counting circuit. By this means, the motor speed could be measured to the nearest $0.1 \mathrm{~Hz}$.

According to Equation (3.7), the amplitude $\mathrm{I}_{0} \cos ^{2}\left(\theta_{1}\right)$ of the sinusoidal stimulus is a function of $\theta_{1}$, the angle between polarizers $P_{1}$ and $P_{4}$. Since $P_{1}$ and $P_{2}$ were fixed perpendicularly, changing the angle between $\mathrm{P}_{1}$ and $\mathrm{P}_{4}$ caused the amplitude of the stimulus to be changed from $0 \%\left(\theta_{1}=90^{\circ}\right)$ to $100 \%\left(\theta_{1}=0^{0}\right.$ or $\left.180^{\circ}\right)$ of the average value.

In order to be able to determine the relative phase between the test flash and the sinusoidal stimulus, the latter was monitored by a silicon photodiode detector (Ealing model \#28-8209) placed next to the beam splitter $\mathrm{BS}_{1}$ as shown in Fig. 3.1. The detector was connected to an oscilloscope in order to display the waveform. (Depending on the reflection/transmission properties of $\mathrm{BS}_{1}$, the signal received by the detector was generally different from that received by the subject's eye. However this did not hinder the function of the detector described below.) Also fed to the detector via a fiber-optic cable, F.O.C., was an optical pulse sampled from the flash-tube $S_{3}$. The appearance on the oscilloscope was similar to that sketched in Fig. 1.6(a). By adjusting the flash delay, the pulse could be positioned at any phase of the sinusoidal stimulus with an accuracy of about $\pm 2^{\circ}$. 


\subsection{Adjustments and calibrations}

\section{AC/DC beam balance}

In order to ensure that rotation of $\mathrm{P}_{4}$ altered only the amplitude modulation, and not the average value of the stimulus, it was necessary to equate the intensity of beam $B$ with the average intensity of beam $A . \mathrm{P}_{0}$ was first oriented to transmit this average intensity. Using carefully positioned opaque screens in beams $A$ and $B$, a bipartite field of view was achieved with the left and right halves illuminated by $A$ and $B$ respectively. $\mathrm{P}_{3}$ was then rotated (relative to $P_{2}$ ) until the two halves appeared to be matched in intensity. With the screens removed, $\mathrm{P}_{0}$ was set into rotation so that the stimulus frequency exceeded the C.F.F. Increment thresholds were then determined for different orientations of $\mathrm{P}_{4}$. No significant differences were observed indicating that the $\mathrm{AC}$ and DC beams were balanced.

\section{Modulation control}

The percentage modulation, $\mathrm{M}$, of the stimulus is the ratio of the amplitude of the sinusoidal input to its average value. In terms of the peak and trough intensities, $\mathrm{L}_{\max }$ and $\mathrm{L}_{\min }, \mathrm{M}$ is given by

$$
\mathrm{M}=\left(\mathrm{L}_{\max }-\mathrm{L}_{\min }\right) /\left(\mathrm{L}_{\max }+\mathrm{L}_{\min }\right)
$$

$\mathrm{L}_{\max }$ and $\mathrm{L}_{\min }$ were determined in this experiment using an Ealing Research Photometer in conjunction with a broad band silicon detector placed at the pupil, X. For different orientations of $\mathrm{P}_{4}, \mathrm{~L}_{\max }$ and $\mathrm{L}_{\min }$ were obtained as the maximum and minimum 
photometer readings observed during rotation of $\mathrm{P}_{0}$. The relationship between the percent modulation and the orientation of the polarizer $\mathrm{P}_{4}$ is shown in Fig. 3.3.

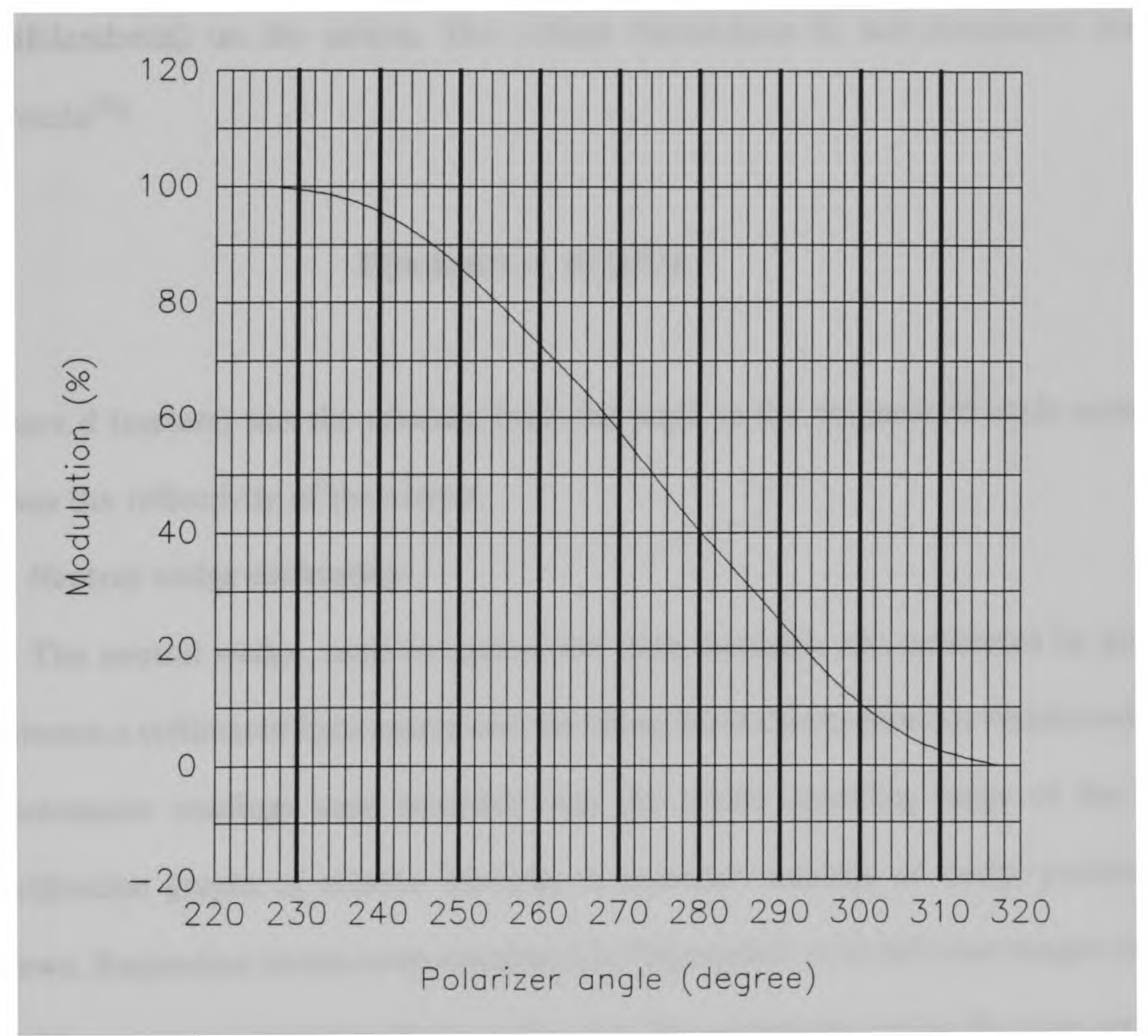

Figure 3.3 Modulation as a function of orientation of polarizer $\mathrm{P}_{4}$ 


\section{Retinal illuminance}

The average retinal illuminance produced by the visual signal generator was measured by placing a magnesium oxide coated screen a few centimeters behind the artificial pupil, X. A Minolta spotmeter was used to measure the luminance L (millilamberts) on the screen. The retinal illuminance E, was calculated from the formula ${ }^{[41]}$ :

$$
\mathrm{E}(\text { troland })=10^{7} \mathrm{Ld}^{2} / \mathrm{r}
$$

where $d$ (meters) was the distance from the pupil to the magnesium oxide screen and $\mathrm{r}$ was the reflectivity of the screen.

\section{Neutral wedge calibration}

The neutral wedge, used to control the flash intensity, was calibrated by placing it between a collimated light source and the broad band silicon detector mentioned above. Photometer readings were recorded over the whole operating range of the wedge. Calibration graphs of relative intensity vs recorder readings of wedge position were drawn. Regression curves were calculated by "Sigmaplot" to fit different ranges of wedge position. A typical graph is shown in Fig. 3.4. The parameters of the fit curve were used in subsequent calculations of relative intensity for a given wedge setting. 


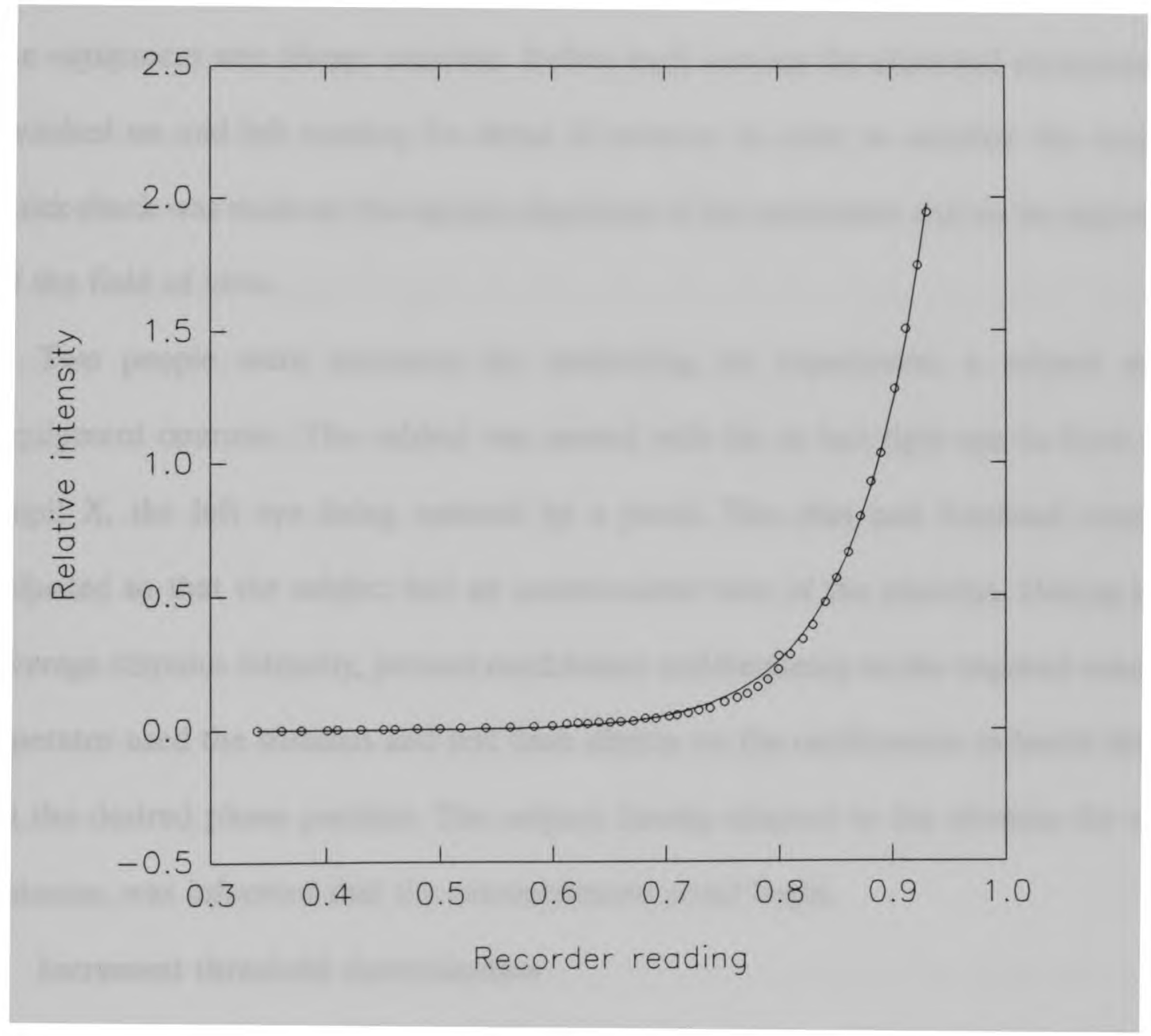

Figure 3.4 Relative intensity vs recorder readings of wedge position 


\subsection{Experimental procedures}

\section{General procedure}

A regular schedule was followed for ensuring that the alignment and calibration of the equipment was always accurate. Before each session the electrical equipment was switched on and left running for about 15 minutes in order to stabilize the output. A quick check was made on the optical alignment of the equipment and on the appearance of the field of view.

Two people were necessary for conducting an experiment; a subject and an equipment operator. The subject was seated with his or her right eye in front of the pupil $\mathrm{X}$, the left eye being covered by a patch. The chin and forehead rests were adjusted so that the subject had an unobstructed view of the stimulus. Having set the average stimulus intensity, percent modulation and frequency to the required values, the operator used the stimulus and test flash display on the oscilloscope to locate the flash at the desired phase position. The subject, having adapted to the stimulus for several minutes, was informed that the measurements could begin.

\section{Increment threshold determination}

The majority of experiments in this study required measurements of the threshold intensity of the test flash when it was superimposed on a bright flickering background. In preliminary trials it was found that observer fatigue set in after about one hour depending on the subject and the experimental conditions. This tended to increase both the time per reading and the uncertainty associated with a single reading.

With this in mind, the following relatively quick procedure was adopted for threshold 
determinations and was found to yield acceptably accurate and repeatable results. For each reading the subject first adjusted the neutral density wedge until the flash was always visible (there was an audible click associated with each flash-tube discharge). The subject then decreased the flash intensity very slowly until the flash was visible on about $50 \%$ of its presentations. To increase or decrease the test flash intensity, the subject pushed either of the two push buttons which actuated the wedge stepper motor. Operation of the stepper motor was also clearly audible; thus the subject could be confident that the wedge had responded to the buttons being pushed.

In order to obtain a complete output waveform for a given input stimulus, the operator set the test flash at each of eight different phase positions relative to the input stimulus; $0^{\circ}, 45^{\circ}, 90^{\circ}, 135^{\circ}, 180^{\circ}, 225^{\circ}, 270^{\circ}$, and $315^{\circ}$, but in a random sequence. For each setting, the subject adjusted the test flash to threshold. This whole procedure was then repeated twice more, using different random sequences for the phase positions in each case. Due to apparent increasing subject sensitivity during an experiment, this random sequencing was of paramount importance.

At the end of each session, which lasted for about one hour, the threshold intensity for an effective zero percent modulation amplitude was determined by setting the stimulus frequency to well above the fusion frequency. The main reason for doing this was to determine whether the subject's overall sensitivity varied from day to day. It would also tend to reveal equipment problems such as optical misalignment. 


\section{Chapter 4. Results, Calculations and Discussion}

\subsection{Output Waveform}

For a linear system, a sinusoidal input results in a sinusoidal output. It was therefore of interest to determine whether the measured output showed any signs of distortion. Such was the case in an earlier study ${ }^{[15,18,35]}$ using the same basic techniques. The type of distortion is illustrated in Fig. 4.1. However, this may have been due to the way the data was collected. In that study, three threshold determinations were made with the flash temporally positioned at $0^{\circ}$ on the input stimulus, then at $45^{\circ}$, then at $90^{\circ}$, etc. In other words the settings were not randomized. In the present study, as noted earlier, increasing subject sensitivity in the course of an experiment resulted in a corresponding lowering of thresholds. Fig. 4.2 shows how this would affect results taken in the previous study. The upper curve is a true sinusoid. The lower one is the same curve from which a factor is subtracted of the form $\mathrm{I}=\mathrm{Ae}^{-\mathrm{c} \phi}$, where $\phi$ is the phase angle and $\mathrm{A}$ and $\mathrm{c}$ are constants. In common with Fig. 4.1, this curve has an apparent period exceeding $360^{\circ}$. By randomizing the phase settings for the present study, as described earlier, this problem was not anticipated. 


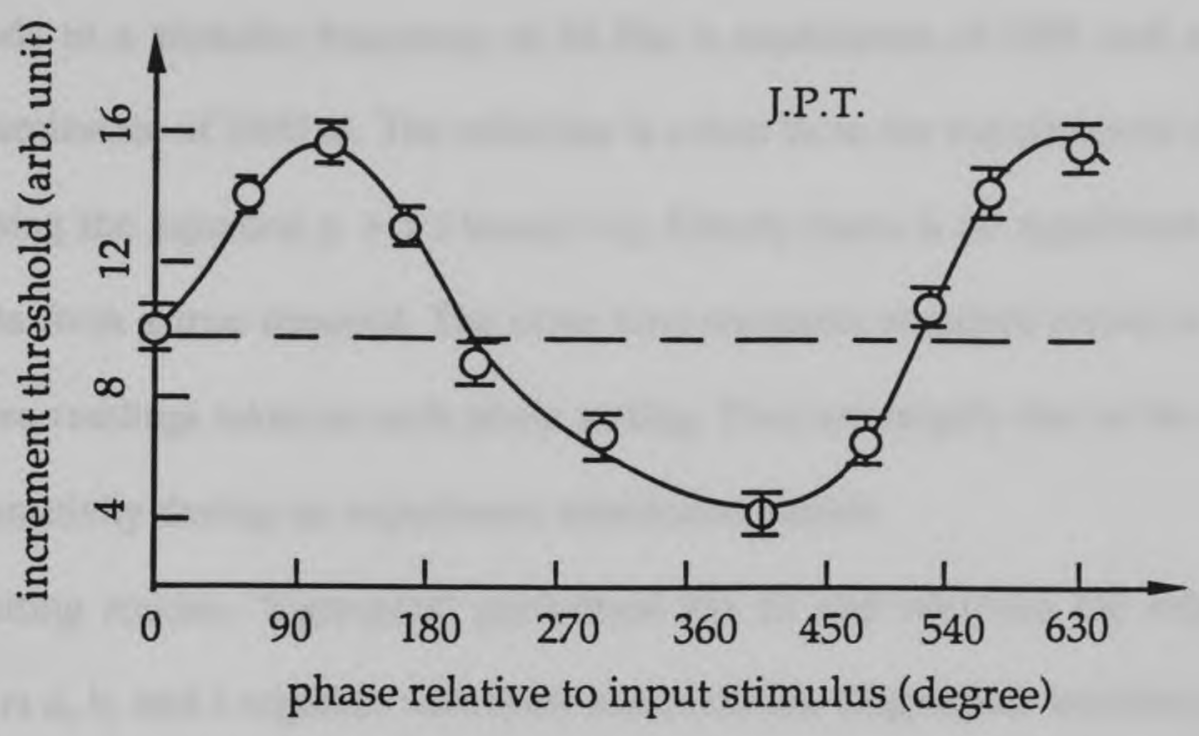

Figure 4.1 Distorted output waveform $(F=25 \mathrm{~Hz})$

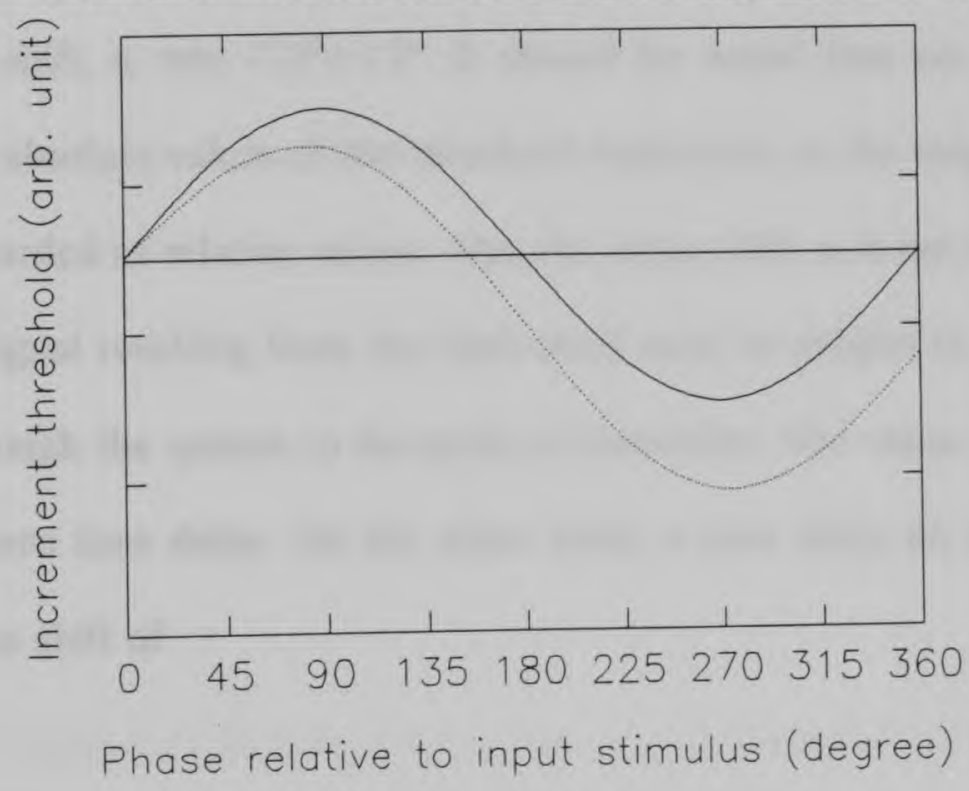

Figure 4.2 True sinusoid (upper curve) distorted sinusoid (lower curve) 
An example of an output waveform for the present study is shown in fig. 4.3(a). It corresponds to a stimulus frequency of $30 \mathrm{~Hz}$, a modulation of $60 \%$ and an average retinal illuminance of $2000 \mathrm{td}$. The solid line is a best fit to the experimental data (open circles) using the equation $y=a+b \cos (c+x)$. Clearly there is no significant distortion of the data from a true sinusoid. The error bars represent standard errors in the mean of the three readings taken at each phase setting. They are largely due to the increasing subject sensitivity during an experiment mentioned earlier.

The fitting routine "Sigmaplot" performed the fit and returned the values of the parameters $\mathrm{a}, \mathrm{b}$, and $\mathrm{c}$ together with their uncertainties. (Sigmaplot searches parameter space using the Marquardt algorithm to minimize $\chi^{2}$.) For the curve in Fig. 4.3 (a) the DC level represented by a was $(2.039 \pm 0.007) 10^{-3}$, the amplitude, $b$, was $(4.9 \pm 0.1) 10^{-4}$ and the phase shift, $c$, was $-7.3^{\circ} \pm 1.2^{\circ}$. It should be noted that no meaning can be attached to the absolute values of the threshold intensities, so the magnitudes of a and b should be regarded as relative values. Also the phase shift, $c$, is not to be regarded as absolute. The signal resulting from the flash itself must be subject to some time delay as it passes through the system to the point of detection. The value of $\mathrm{c}\left(-7.3^{\circ}\right)$ given here assumes zero time delay. On the other hand, a time delay of, say, $10 \mathrm{~ms}$ would result in a phase shift of

$$
\Phi=-7.3^{\circ}-360^{\circ} \cdot 10 \mathrm{~ms} / 33.3 \mathrm{~ms}=-115.3^{\circ}
$$

The output waveform for input stimulus frequencies of $5,10,15,20,25,35,40$, and 
$45 \mathrm{~Hz}$ (60\% modulation, 2000 td, subject R.A.B.) are displayed in Fig. 4.3 (b).

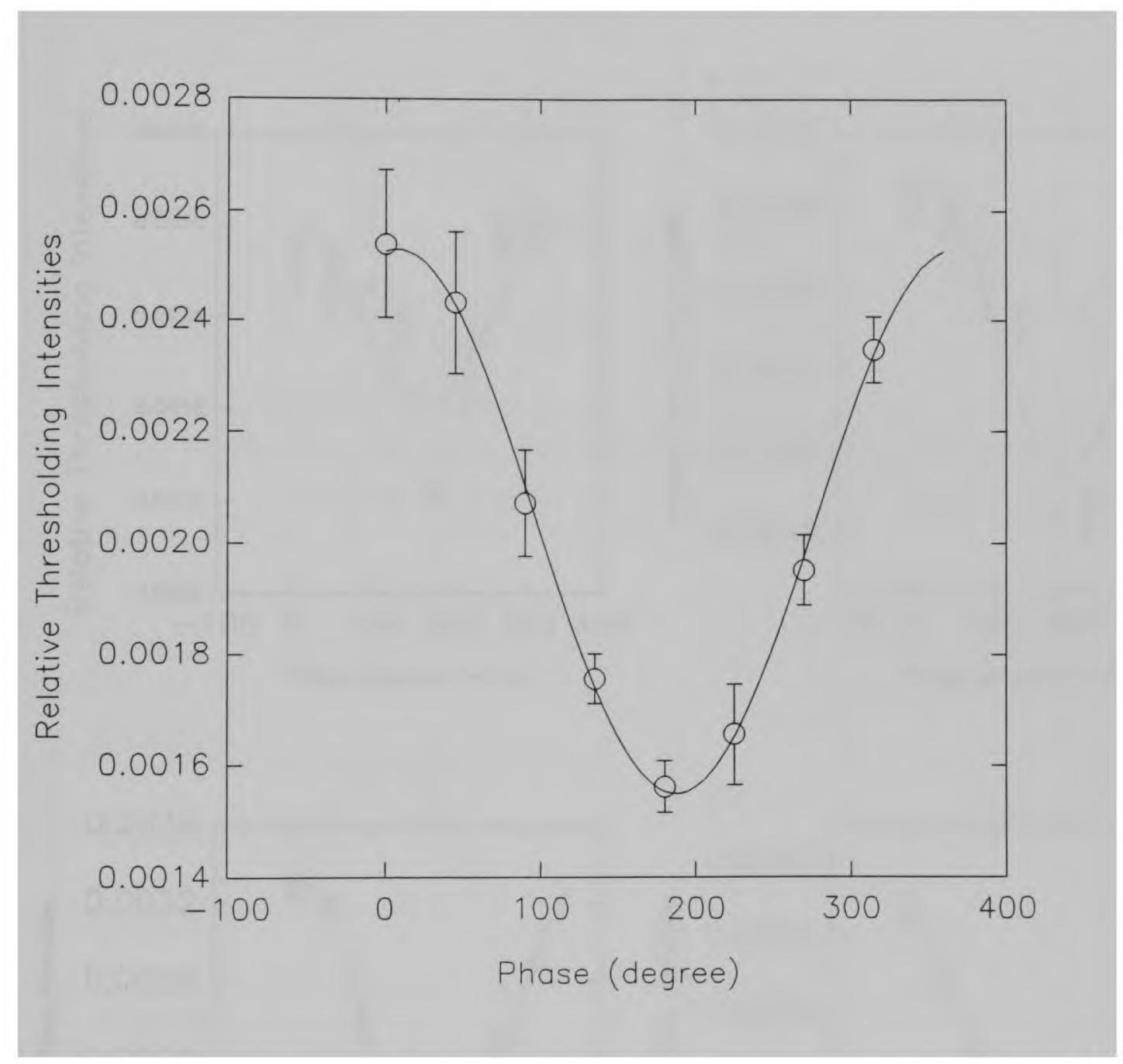

Figure 4.3 (a) Output waveform at $60 \%$ modulation (frequency $=30 \mathrm{~Hz}$ ) 


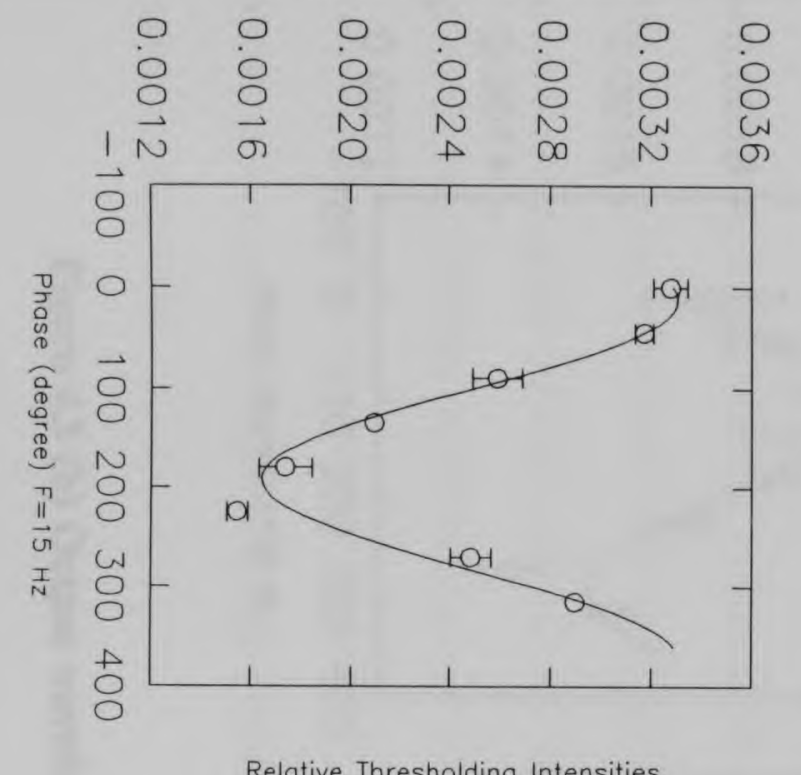

Relative Thresholding Intensities

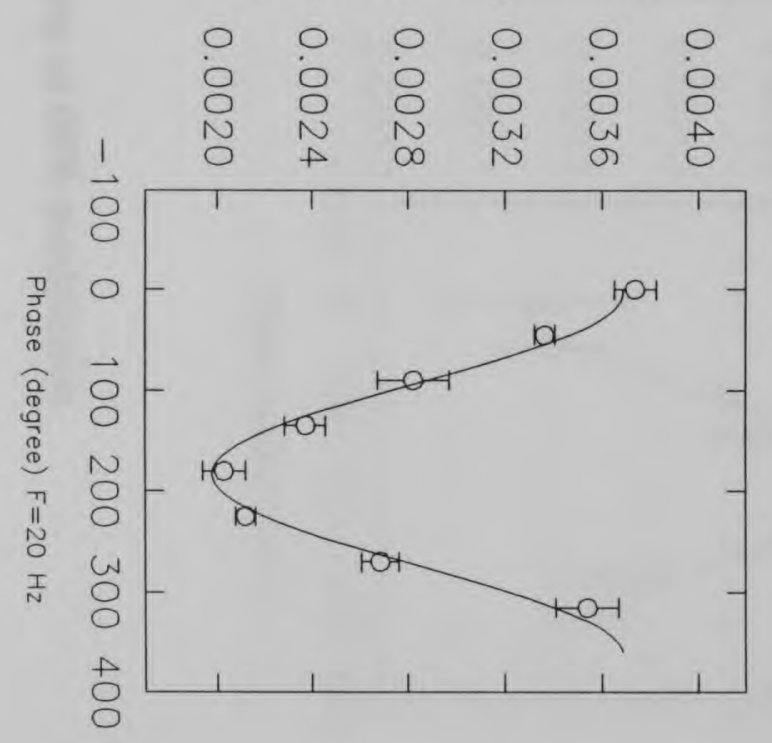

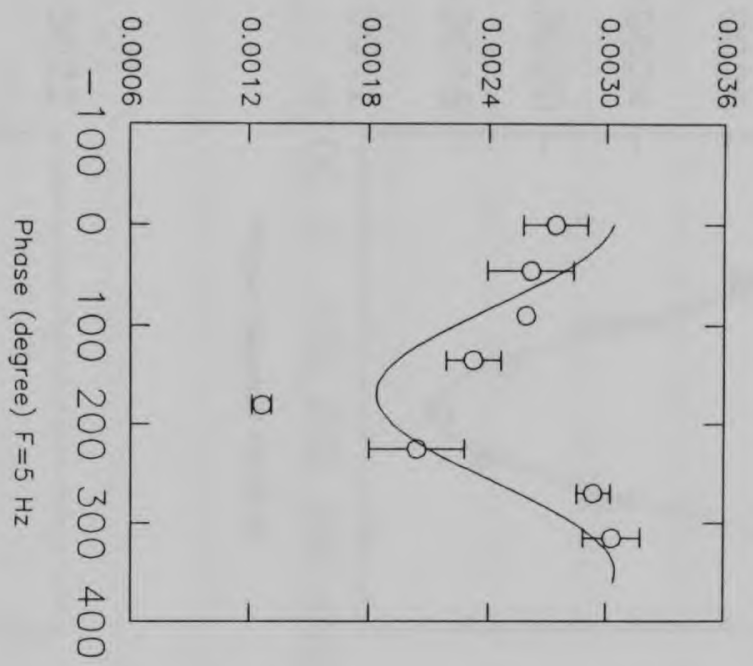

Relative Thresholding Intensities

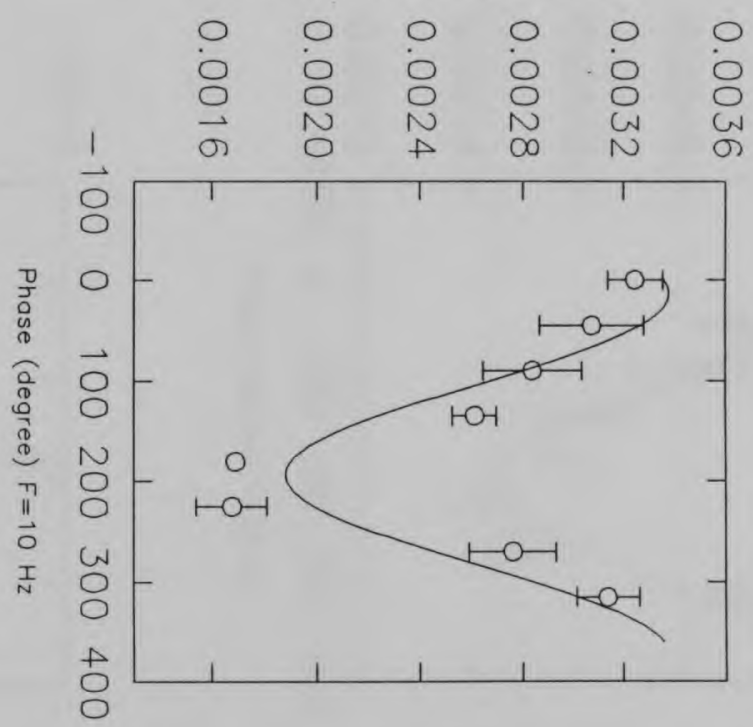



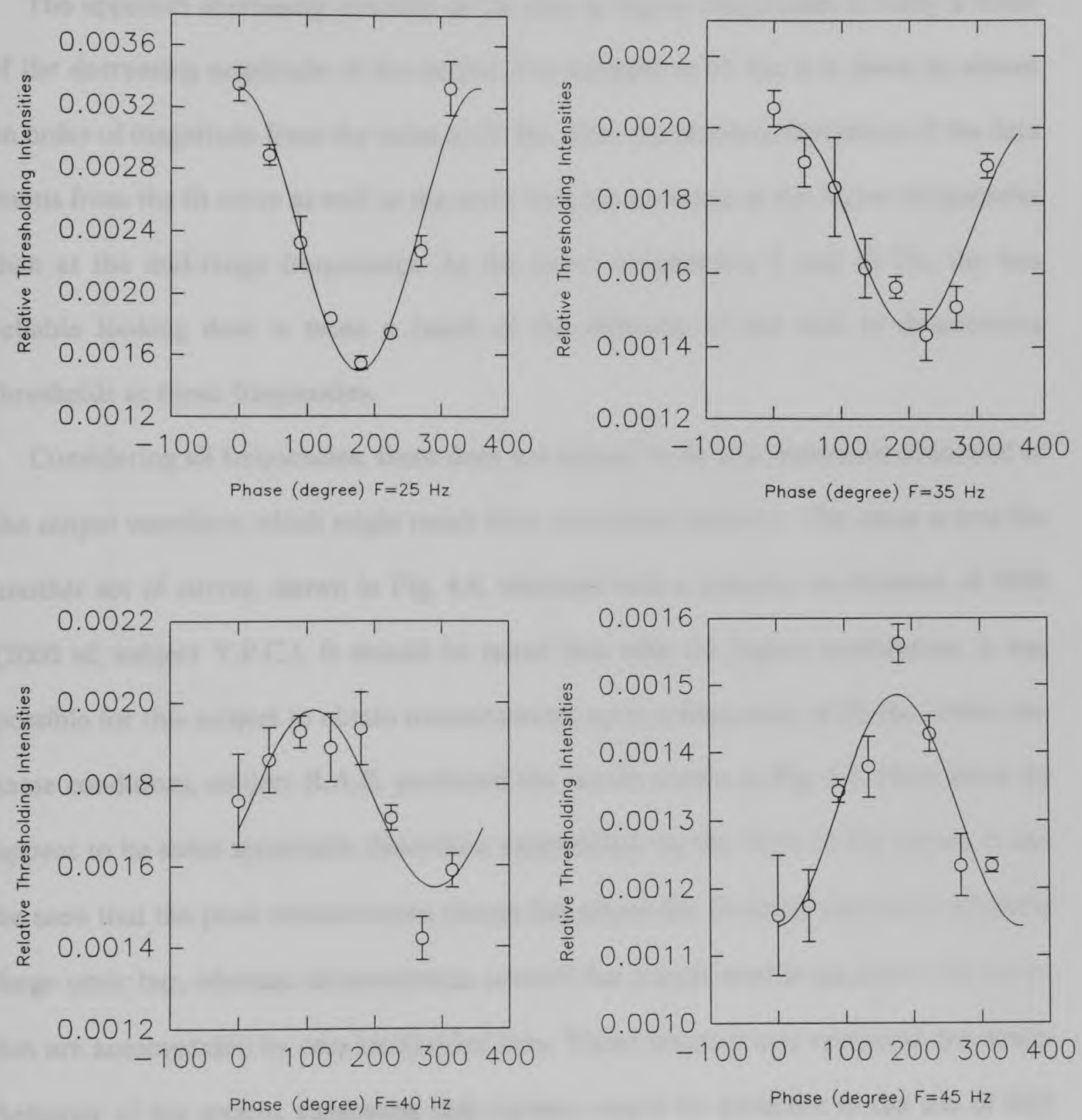

Figure 4.3 (b) Output waveform at $60 \%$ modulation 
The apparent decreasing accuracy of the data at higher frequencies is really a result of the decreasing amplitude of the output. For example at $45 \mathrm{~Hz}$, it is down by almost an order of magnitude from the value at $25 \mathrm{~Hz}$. Thus the absolute deviations of the data points from the fit curve as well as the error bars are no worse at the higher frequencies than at the mid-range frequencies. At the lower frequencies, 5 and $10 \mathrm{~Hz}$, the less reliable looking data is more a result of the difficulty of the task of determining thresholds at these frequencies.

Considering all frequencies, there does not appear to be any systematic distortion of the output waveform which might result from non-linear behavior. The same is true for another set of curves, shown in Fig. 4.4, obtained with a stimulus modulation of $90 \%$ (2000 td, subject Y.P.C.). It should be noted that with the higher modulation, it was possible for this subject to obtain measurements up to a frequency of $50 \mathrm{~Hz}$. Under the same conditions, subject R.A.B. produced the results shown in Fig. 4.5. Here there do appear to be some systematic distortions exemplified by the 10 to $35 \mathrm{~Hz}$ curves. It can be seen that the peak measurement always lies above the fit curve and has a relatively large error bar, whereas measurements around the trough tend to lie above the curve, but are accompanied by very small error bars. These features may represent non-linear behavior of the system, suggesting that caution would be advisable in the use of high stimulus modulations. 


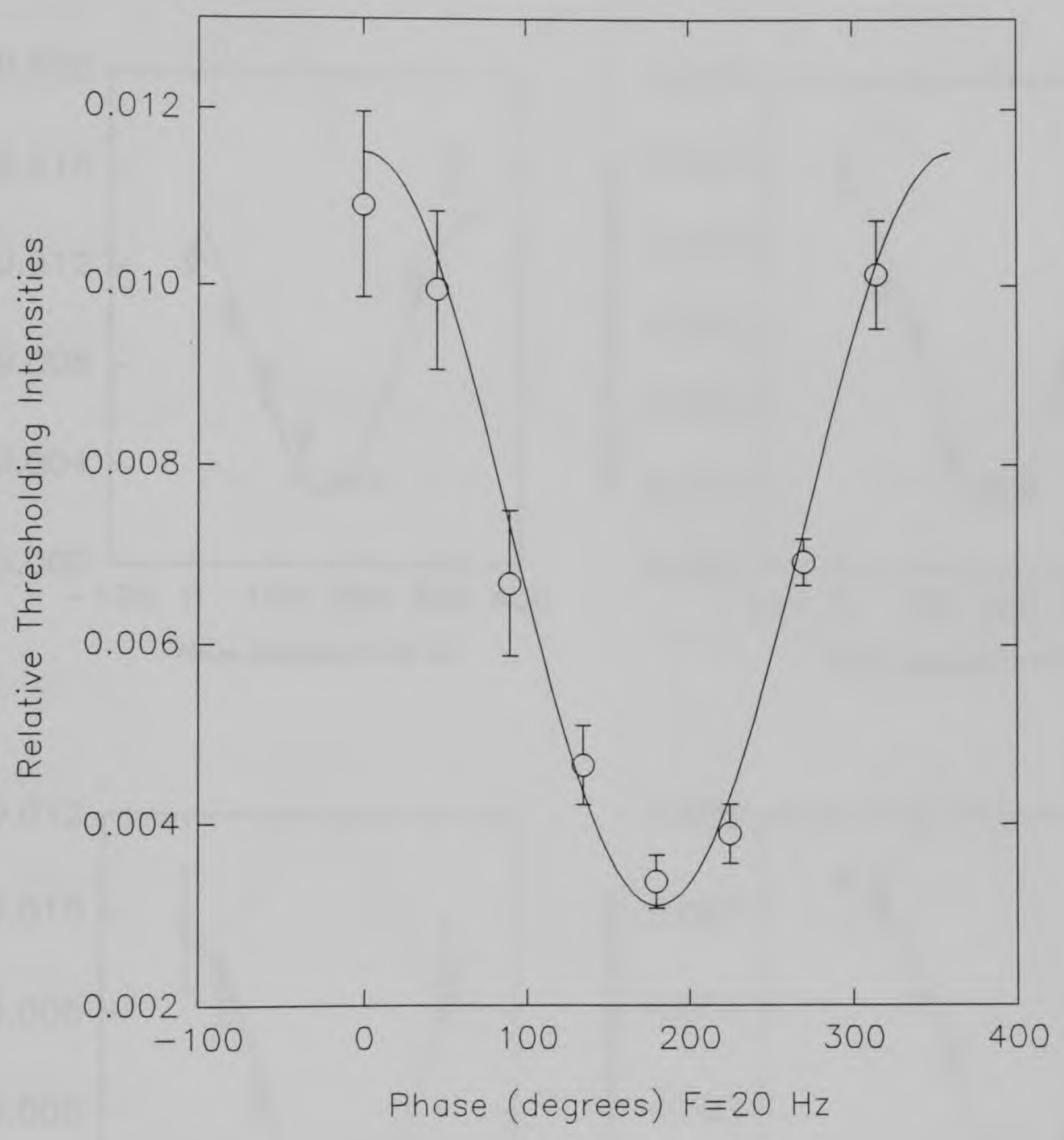

$$
\begin{aligned}
& a=(7.3 \pm 0.3) 10^{-3}, \\
& b=(4.2 \pm .5) 10^{-3}, \\
& c=-0.5^{\circ} \pm 6.3^{\circ} .
\end{aligned}
$$

Figure 4.4 (a) Output waveform for frequency $20 \mathrm{~Hz}$ 
Relative Thresholding Intensities

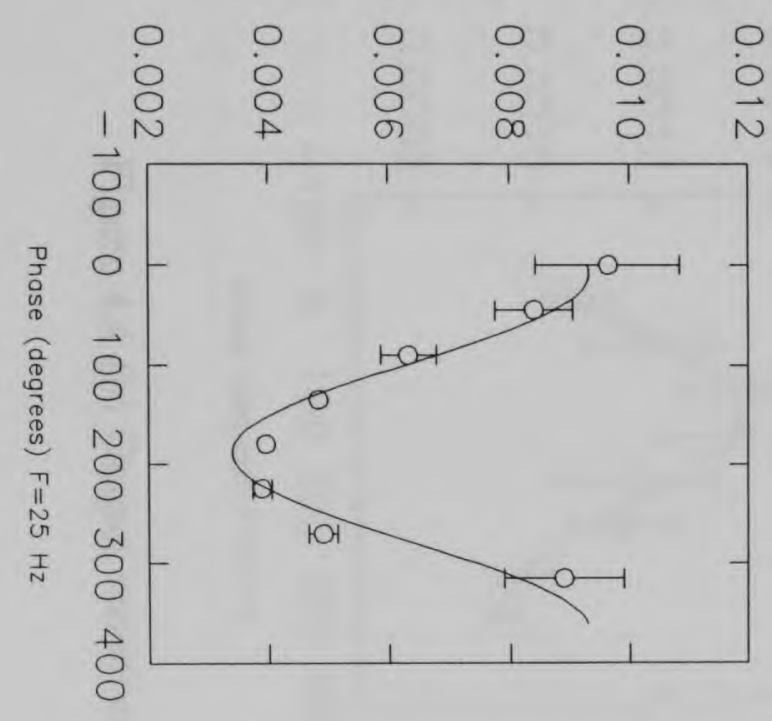

Relative Thresholding Intensities

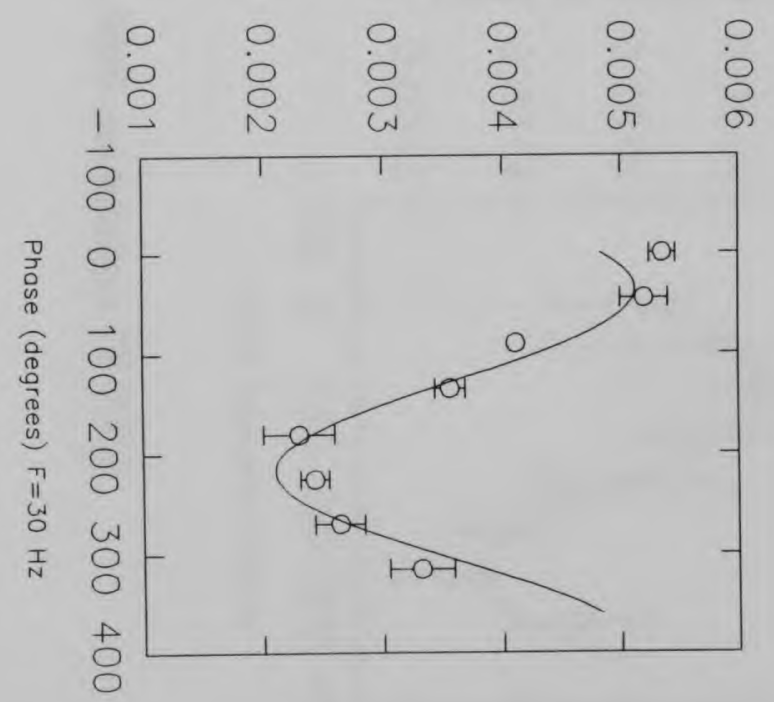

Relative Thresholding Intensities

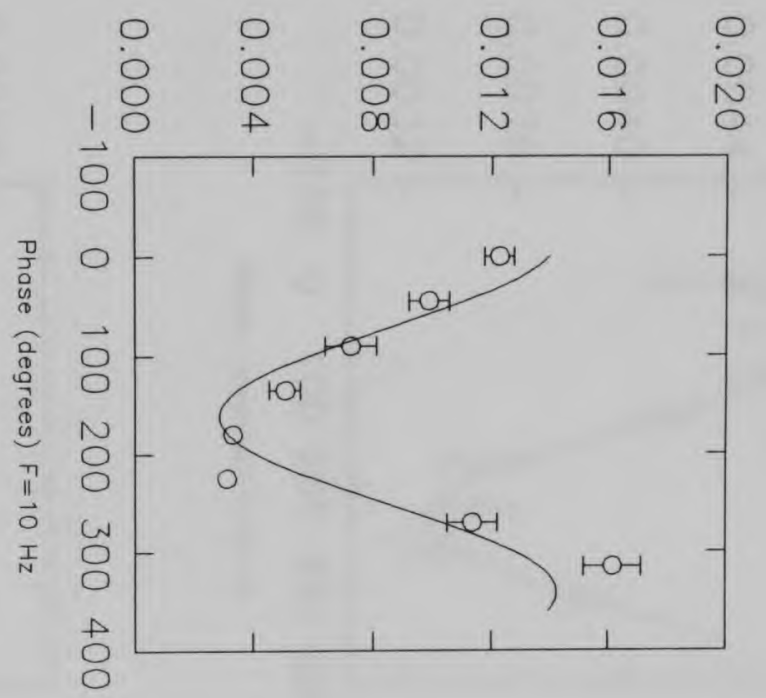

Relative Thresholding Intensities

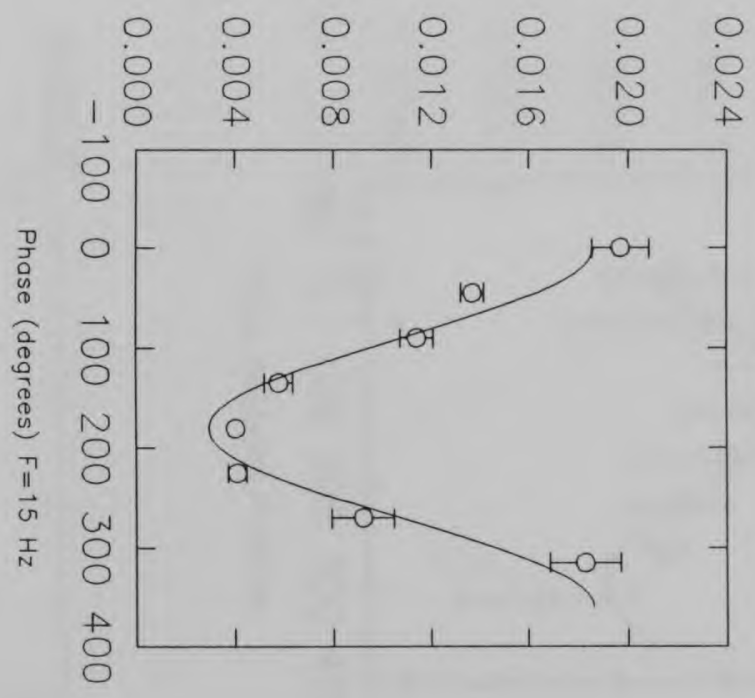



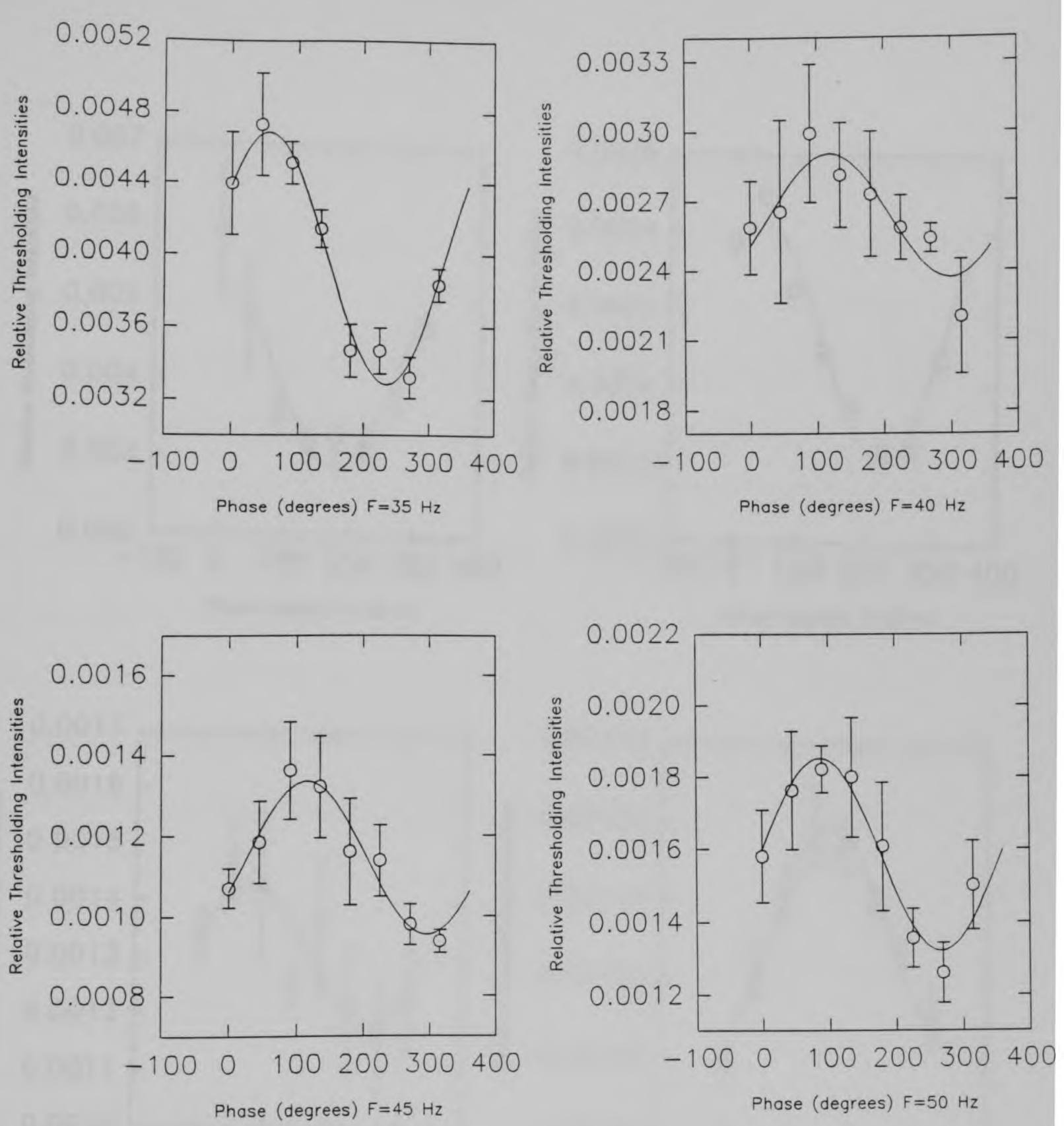

Figure 4.4 (b) Output waveform at $90 \%$ modulation (Y.P.C.) 

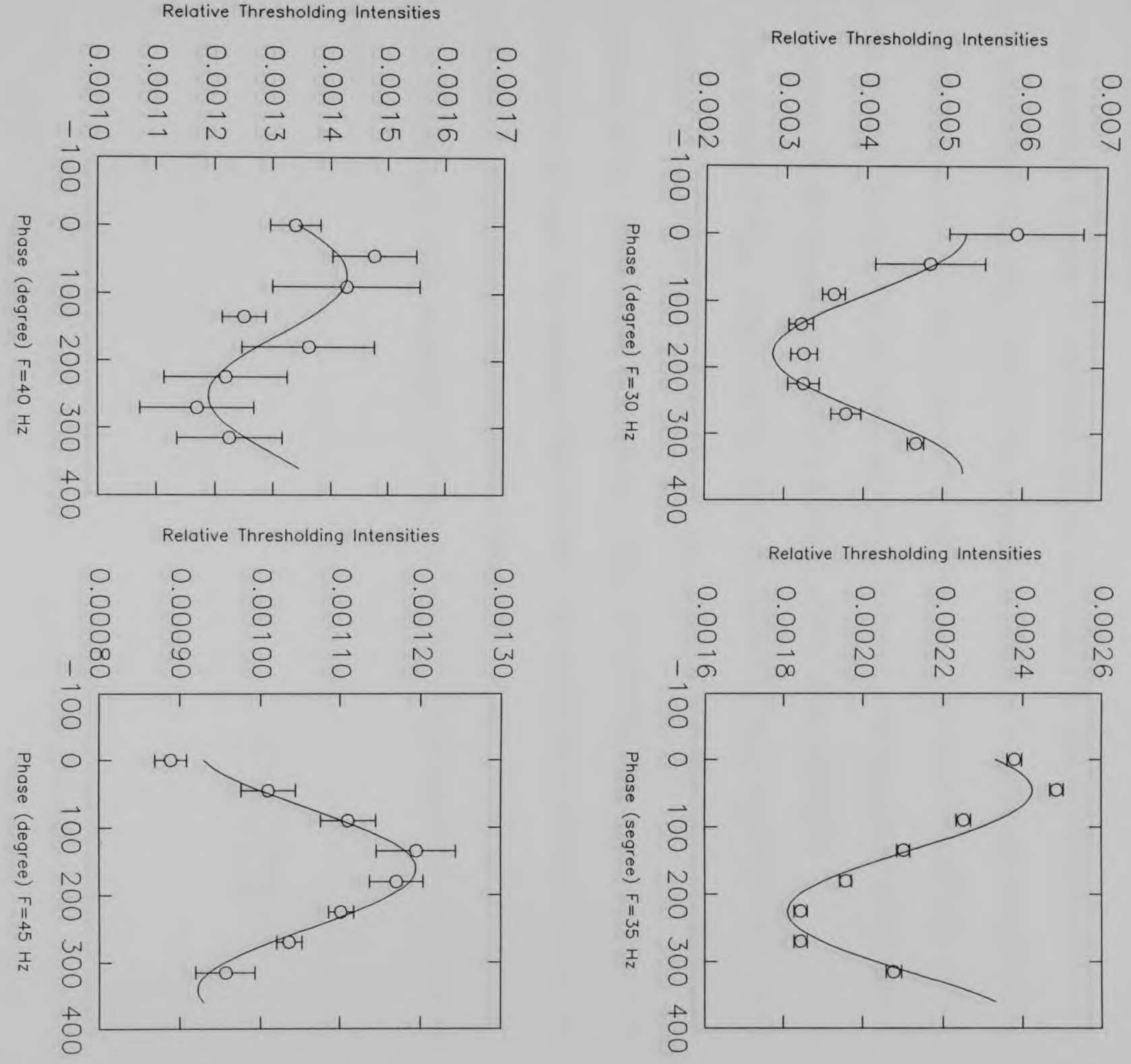

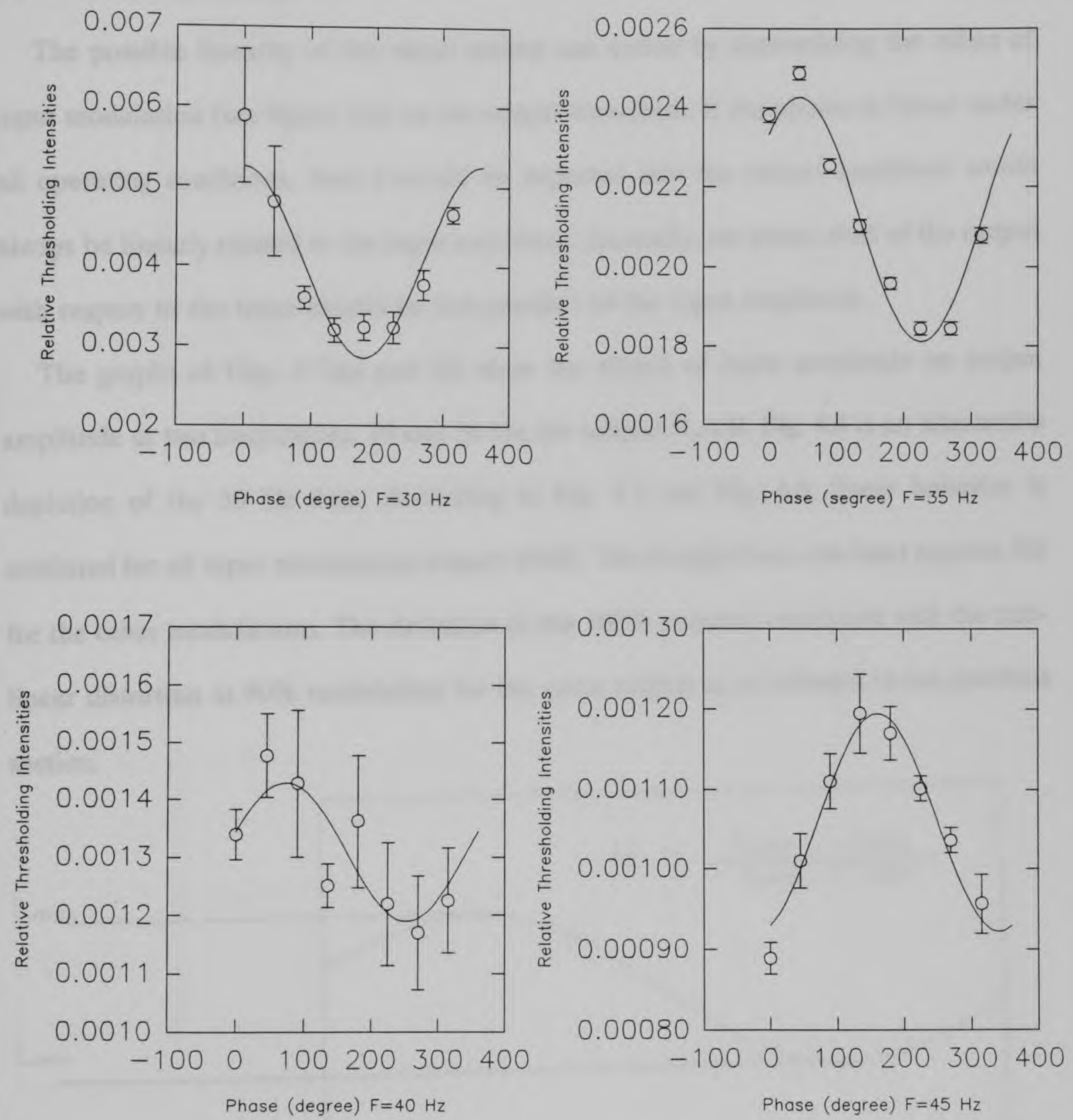

Figure 4.5 Output waveform at $90 \%$ modulation (R.A.B.) 


\subsection{Effects of modulation}

The possible linearity of the visual system was tested by determining the effect of input modulation (see figure 4.6) on the output waveform. If the system is linear under all operating conditions, then it would be expected that the output amplitude would always be linearly related to the input amplitude. Secondly, the phase shift of the output with respect to the input should be independent of the input amplitude.

The graphs of Figs. 4.7(a) and (b) show the effects of input amplitude on output amplitude at two frequencies, 20 and $30 \mathrm{~Hz}$, for subject R.A.B. Fig. 4.8 is an alternative depiction of the $30 \mathrm{~Hz}$ data. According to Fig. 4.7 and Fig. 4.8, linear behavior is exhibited for all input modulations expect $100 \%$. The straight lines are least squares fits for the other modulations. The deviation of the $100 \%$ points is consistent with the nonlinear distortion at $90 \%$ modulation for the same subject as mentioned in the previous section.

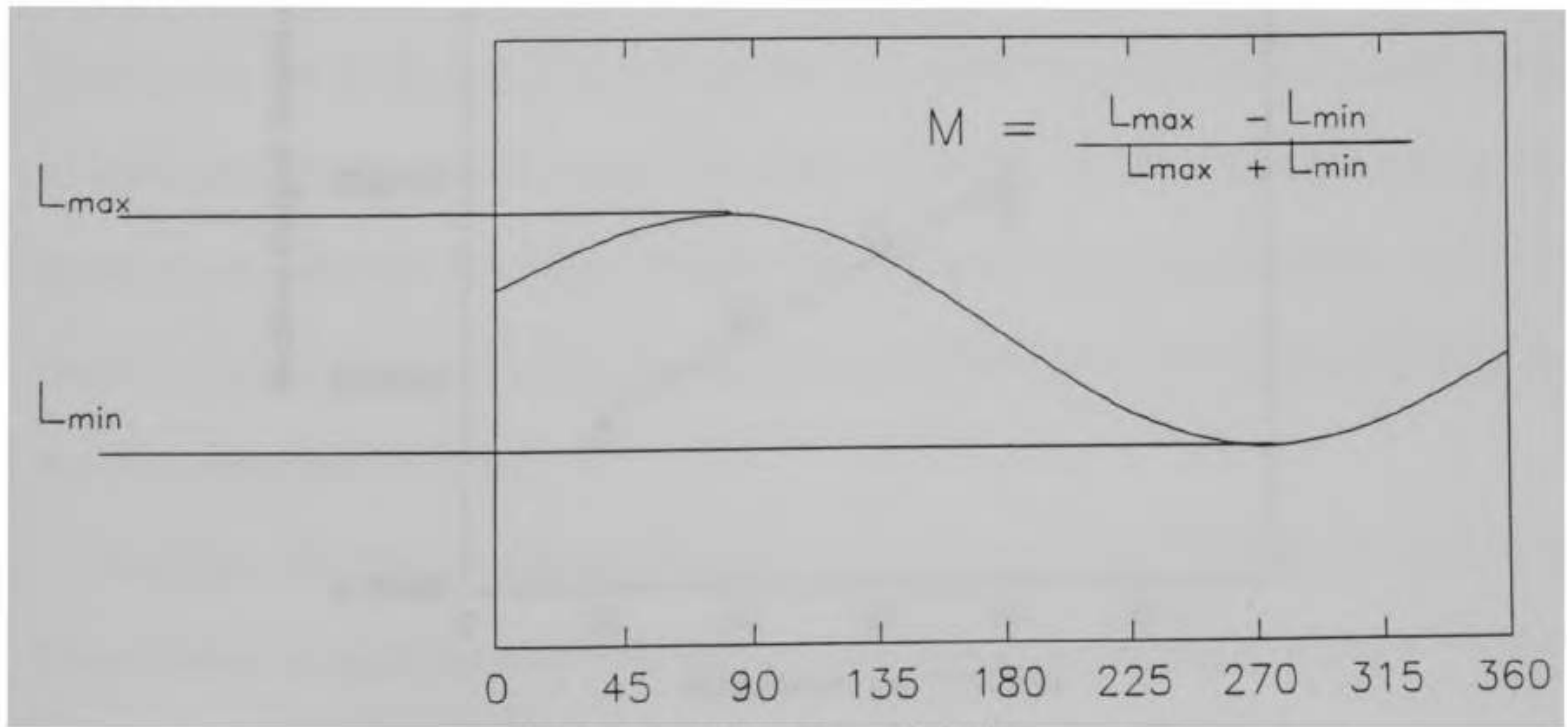

Figure 4.6 Input modulation 

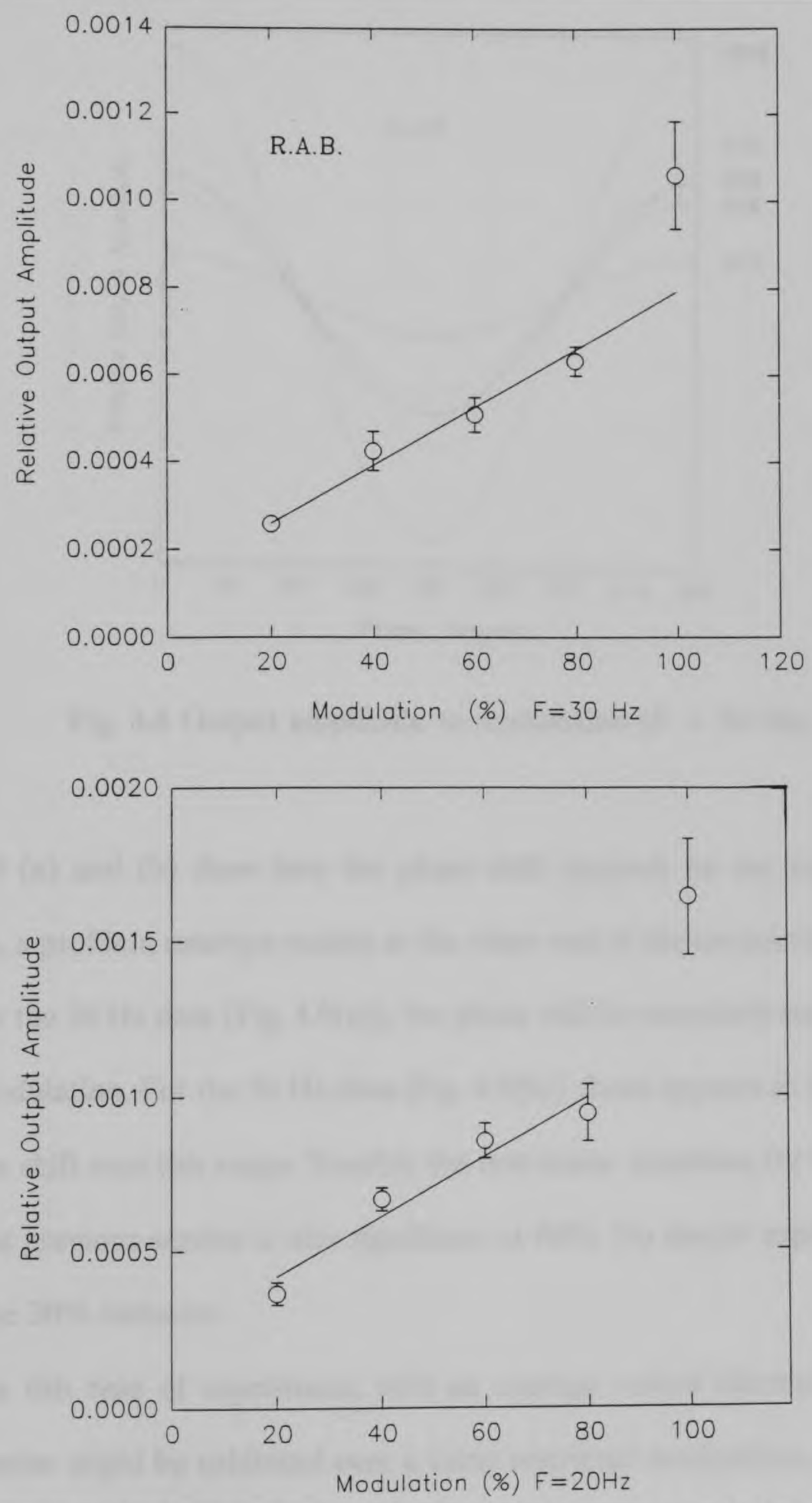

Figure 4.7 Output amplitude as a function of modulation 


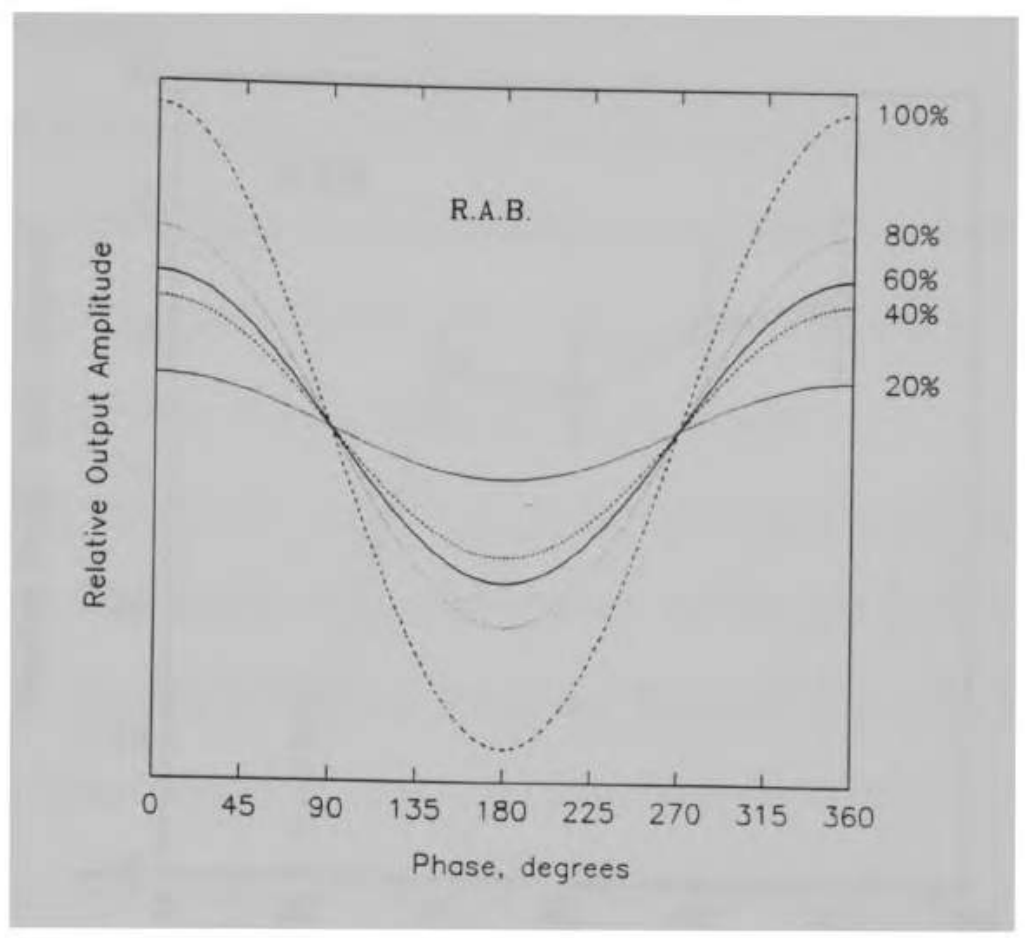

Fig. 4.8 Output amplitude vs modulation $(\mathrm{F}=30 \mathrm{~Hz})$

Figs. 4.9 (a) and (b) show how the phase shift depends on the input modulation. From these, a problem emerges mainly at the other end of the modulation scale, namely at $20 \%$. For the $20 \mathrm{~Hz}$ data (Fig. 4.9(a)), the phase shift is essentially constant from $40 \%$ to $100 \%$ modulation. For the $30 \mathrm{~Hz}$ data (Fig. 4.9(b)), there appears to be some increase in the phase shift over this range. Possibly the non-linear distortion for $90 \%$ modulation noted in the previous section is also significant at $80 \%$. No simple explanation suggests itself for the $20 \%$ behavior.

Thus for this type of experiment, with an average retinal illuminance of $2000 \mathrm{td}$, linear behavior might be exhibited over a fairly restricted modulation range, $\sim 40 \%$ to $70 \%$. 

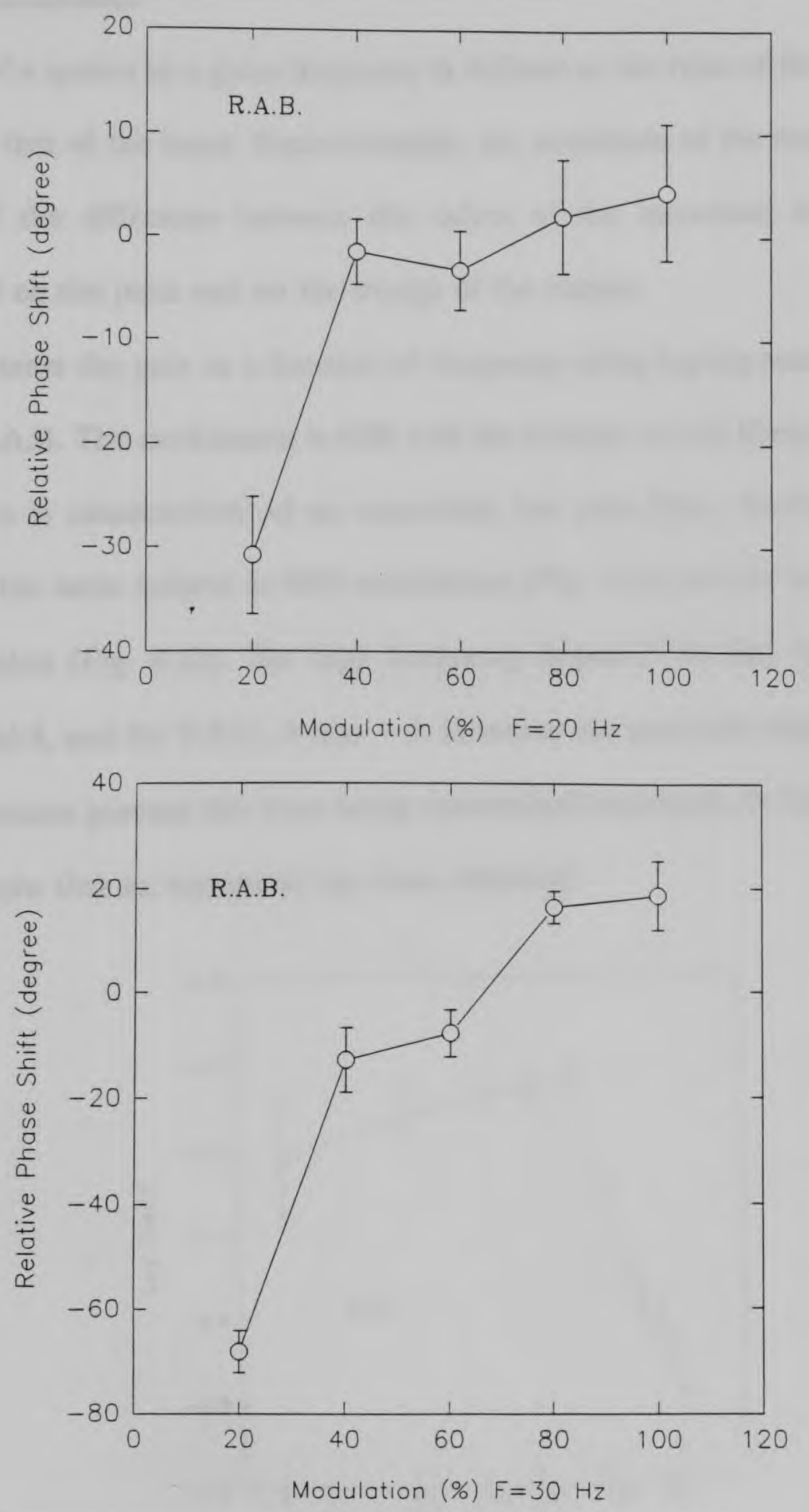

Figure 4.9 Phase shift curves vs modulation 


\subsection{Gain characteristics}

The gain of a system at a given frequency is defined as the ratio of the amplitude of the output to that of the input. Experimentally, the amplitude of the output should be given by half the difference between the values of the increment threshold when superimposed on the peak and on the trough of the output.

Fig. 4.10 shows the gain as a function of frequency using log-log scales (Bode plot) for subject R.A.B. The modulation is $60 \%$ and the average retinal illuminance $2000 \mathrm{td}$. This spectrum is characteristic of an essentially low pass filter. Similar curves were obtained for the same subject at $90 \%$ modulation (Fig. 4.11) and for subject Y.P.C. at 90\% modulation (Fig. 4.12). The high frequency slopes $(>30 \mathrm{~Hz}$ ) for R.A.B. were between 7 and 8, and for Y.P.C., it was $\sim 5$. However the generally larger error bars at higher frequencies prevent this from being determined accurately. In fact it is not clear from the graphs that an asymptote has been obtained.

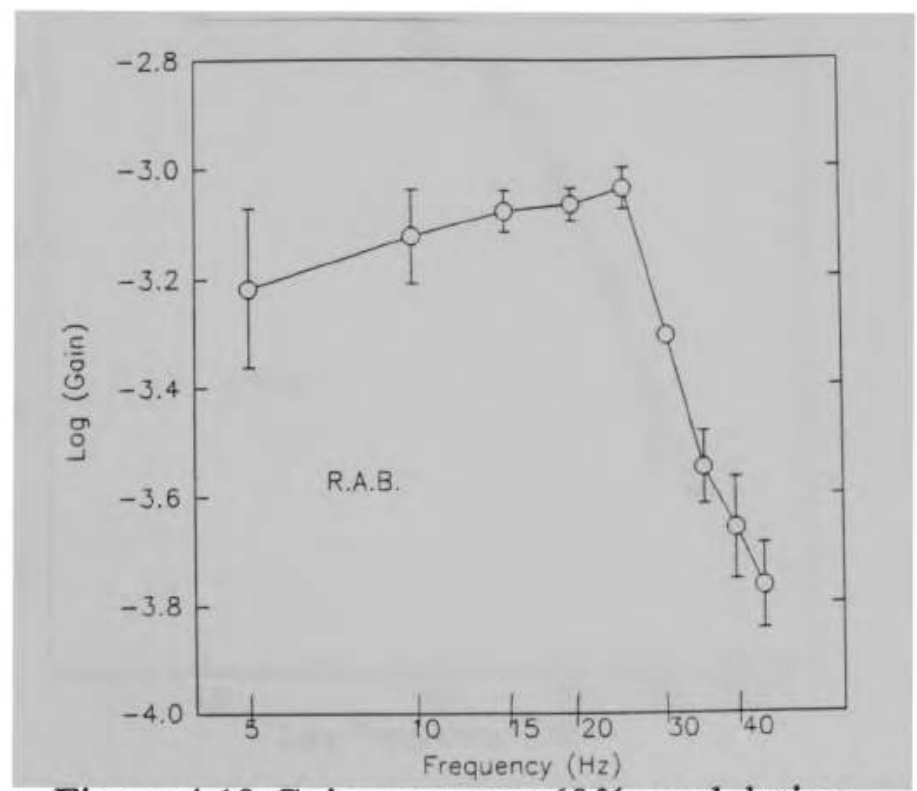

Figure 4.10 Gain curves at $60 \%$ modulation 


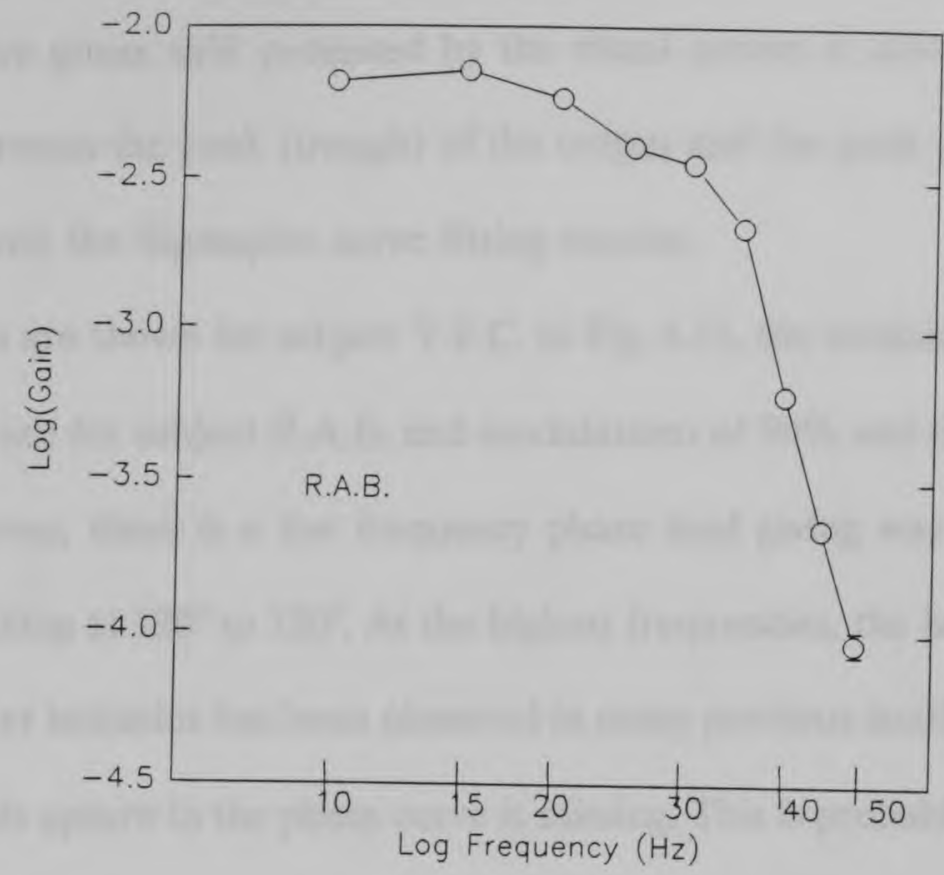

Figure 4.11 Gain curves at $90 \%$ modulation (R.A.B.)

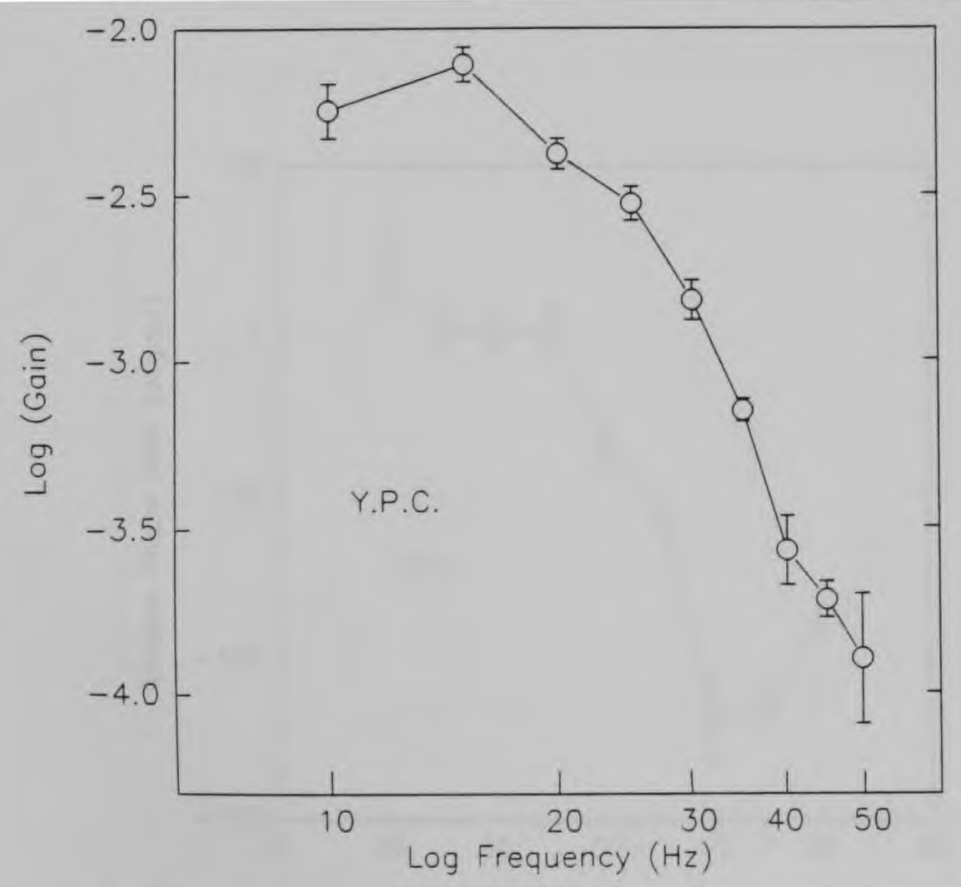

Figure 4.12 Gain curves at $90 \%$ modulation (Y.P.C.) 


\subsection{Phase Shift Characteristics}

The relative phase shift produced by the visual system is determined as the phase difference between the peak (trough) of the output and the peak (trough) of the input, as obtained with the Sigmaplot curve fitting routine.

The results are shown for subject Y.P.C. in Fig. 4.13, the modulation being 90\%. Fig. 4.14 and 4.15 are for subject R.A.B. and modulations of $90 \%$ and $60 \%$ respectively. For both $90 \%$ curves, there is a low frequency phase lead giving way to a high frequency phase lag peaking at $120^{\circ}$ to $150^{\circ}$. At the highest frequencies, the lag decreases to about $100^{\circ}$. This latter behavior has been observed in many previous studies. ${ }^{[14,15,18,28,35]}$ For the $60 \%$ curve, this upturn in the phase curve is missing. This is probably due to the fact that for this modulation, no measurements could be obtained at $50 \mathrm{~Hz}$, the frequency where the effect occurred for this subject when using $90 \%$ modulation.

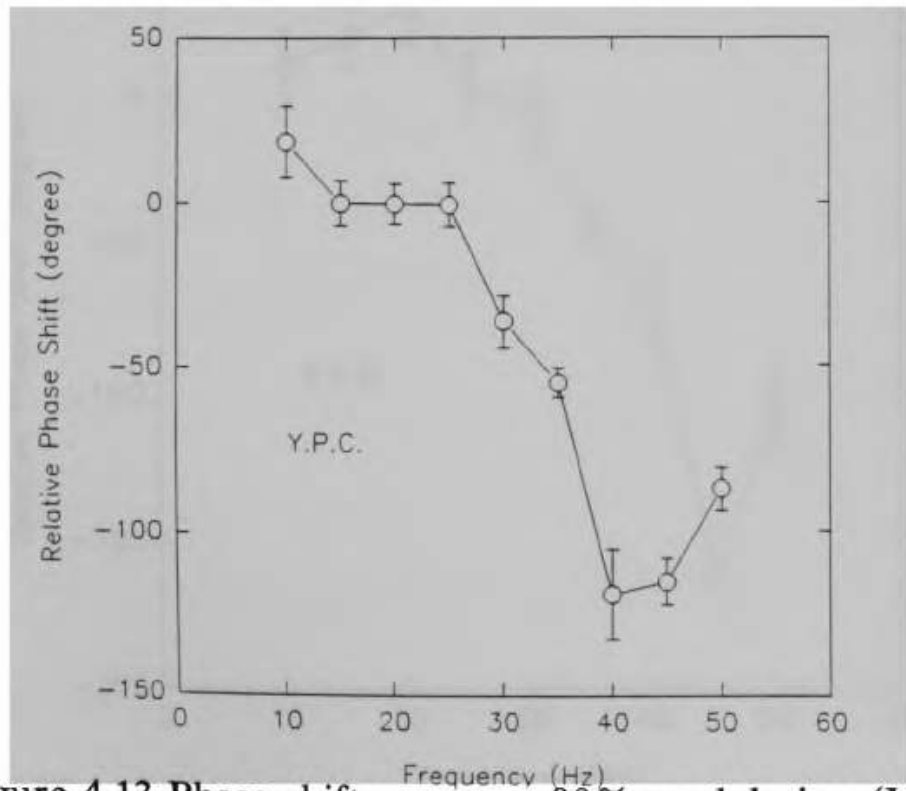

Figure 4.13 Phase shift curves at $90 \%$ modulation (Y.P.C.) 


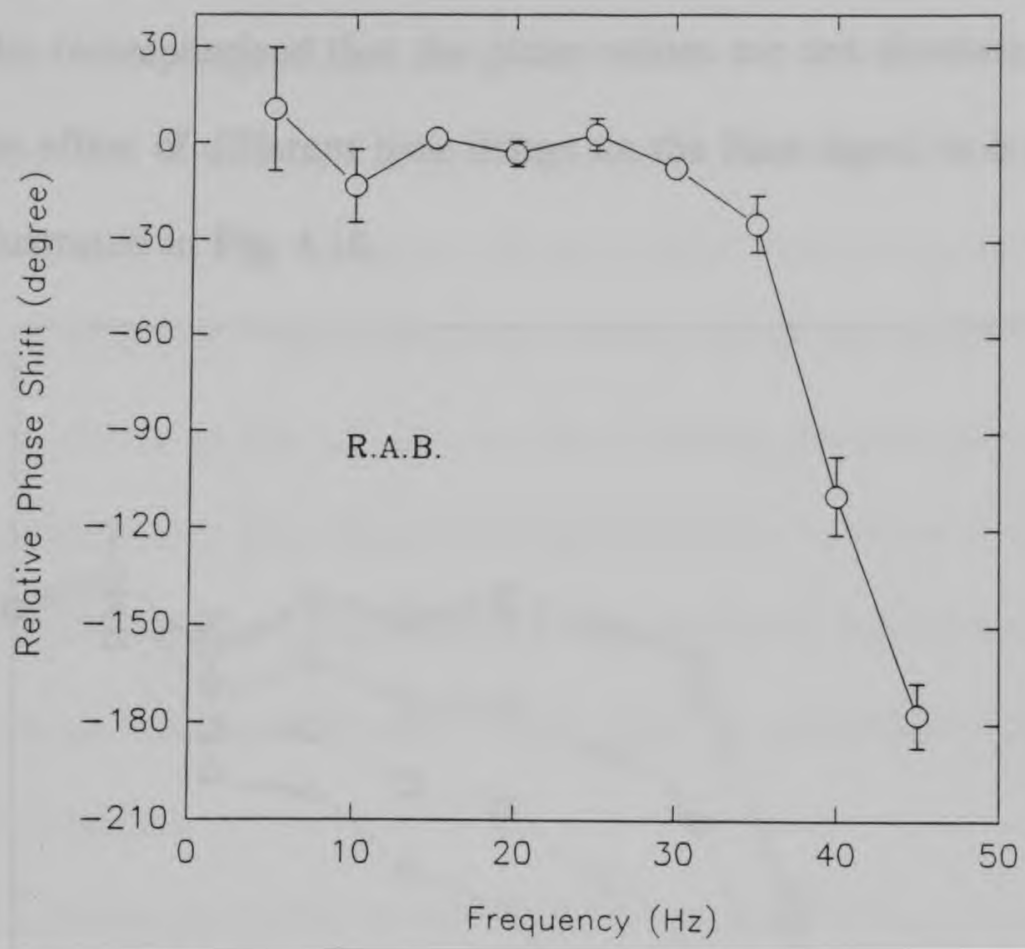

Figure 4.14 Phase shift curves at $90 \%$ modulation (R.A.B.)

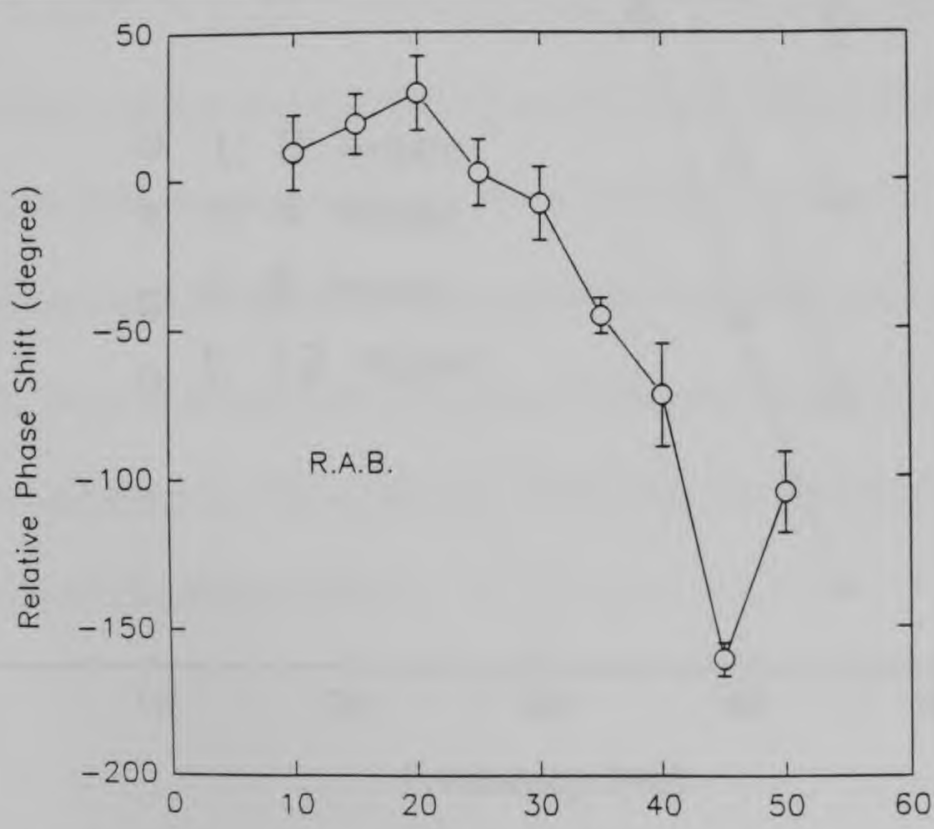

Figure 4.15 Phase shift curves at $60 \%$ modulation 
It must be re-emphasized that the phase values are not absolute, only relative. For example, the effect of different time delays on the flash signal as it passes through the system is illustrated in Fig. 4.16.

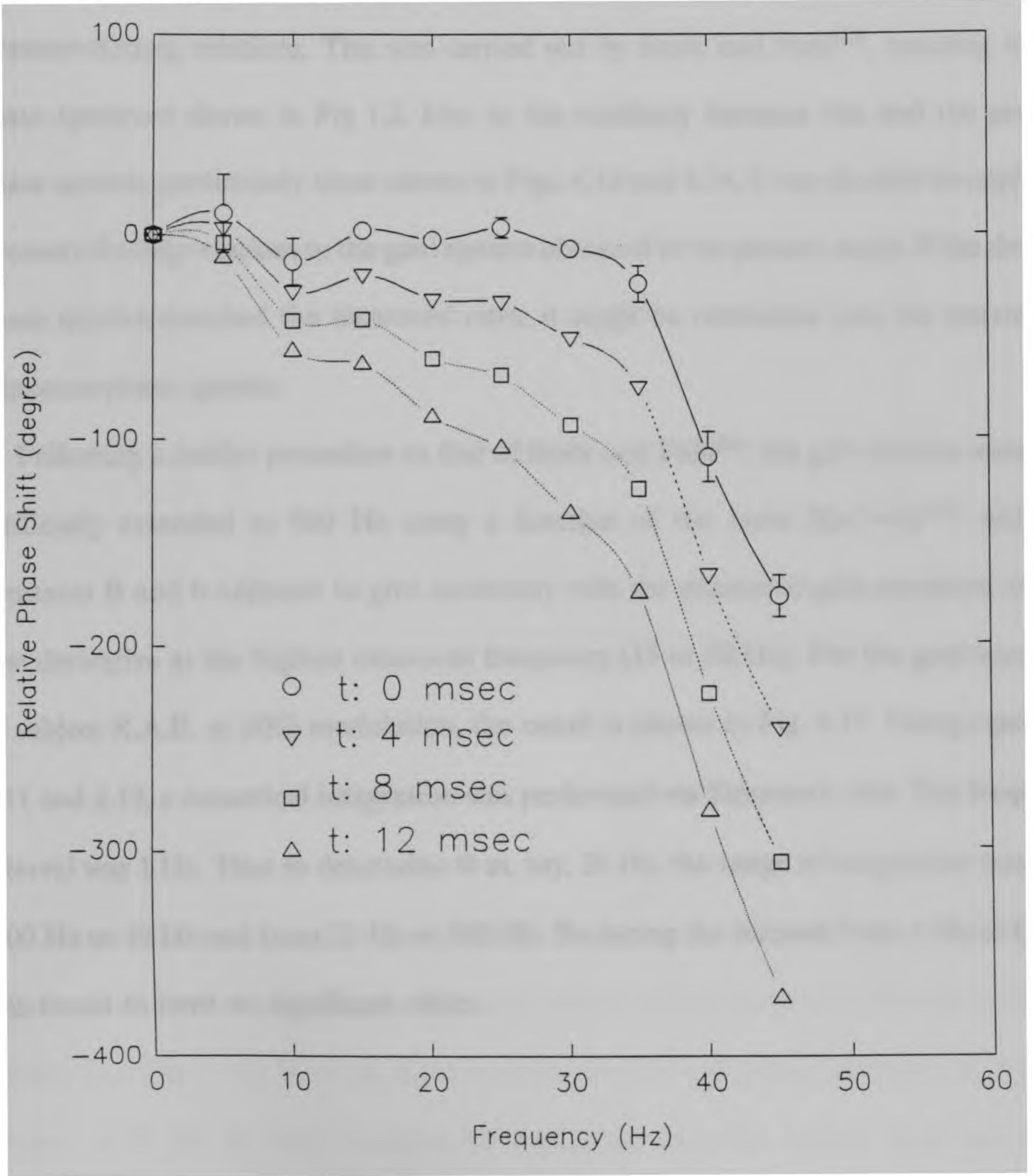

Figure 4.16 Time delay ( $t$ ) correction for the visual system 


\subsection{Relationship between gain and phase}

It was pointed out in 2.3 that if a system is a stable, causal one, and also a minimumphase system, then the phase spectrum can be derived from the gain spectrum via the Kramers-Kronig relations. This was carried out by Stork and Falk ${ }^{[16]}$, resulting in the phase spectrum shown in Fig 1.2. Due to the similarity between this and the present phase spectra, particularly those shown in Figs. 4.13 and 4.14, it was decided to apply the Kramers-Kronig relations to the gain spectra obtained in the present study. If the derived phase spectra matched the measured ones, it might be concluded that the system is a minimum-phase system.

Following a similar procedure to that of Stork and Falk $^{[16]}$, the gain spectra were first artificially extended to $500 \mathrm{~Hz}$ using a function of the form $\mathrm{B}\left(\omega^{4}+\mathrm{b}\right)^{-1 / 2}$, with the constants $\mathrm{B}$ and $\mathrm{b}$ adjusted to give continuity with the measured gain spectrum and its first derivative at the highest measured frequency $(45$ or $50 \mathrm{~Hz})$. For the gain spectrum of subject R.A.B. at $60 \%$ modulation, the result is shown in Fig. 4.17. Using equations 2.11 and 2.12, a numerical integration was performed via Simpson's rule. The frequency interval was $1 \mathrm{~Hz}$. Thus to determine $\Phi$ at, say, $20 \mathrm{~Hz}$, the range of integration was from $-500 \mathrm{~Hz}$ to $19 \mathrm{~Hz}$ and from $21 \mathrm{~Hz}$ to $500 \mathrm{~Hz}$. Reducing the interval from $1 \mathrm{~Hz}$ to $0.1 \mathrm{~Hz}$ was found to have no significant effect. 


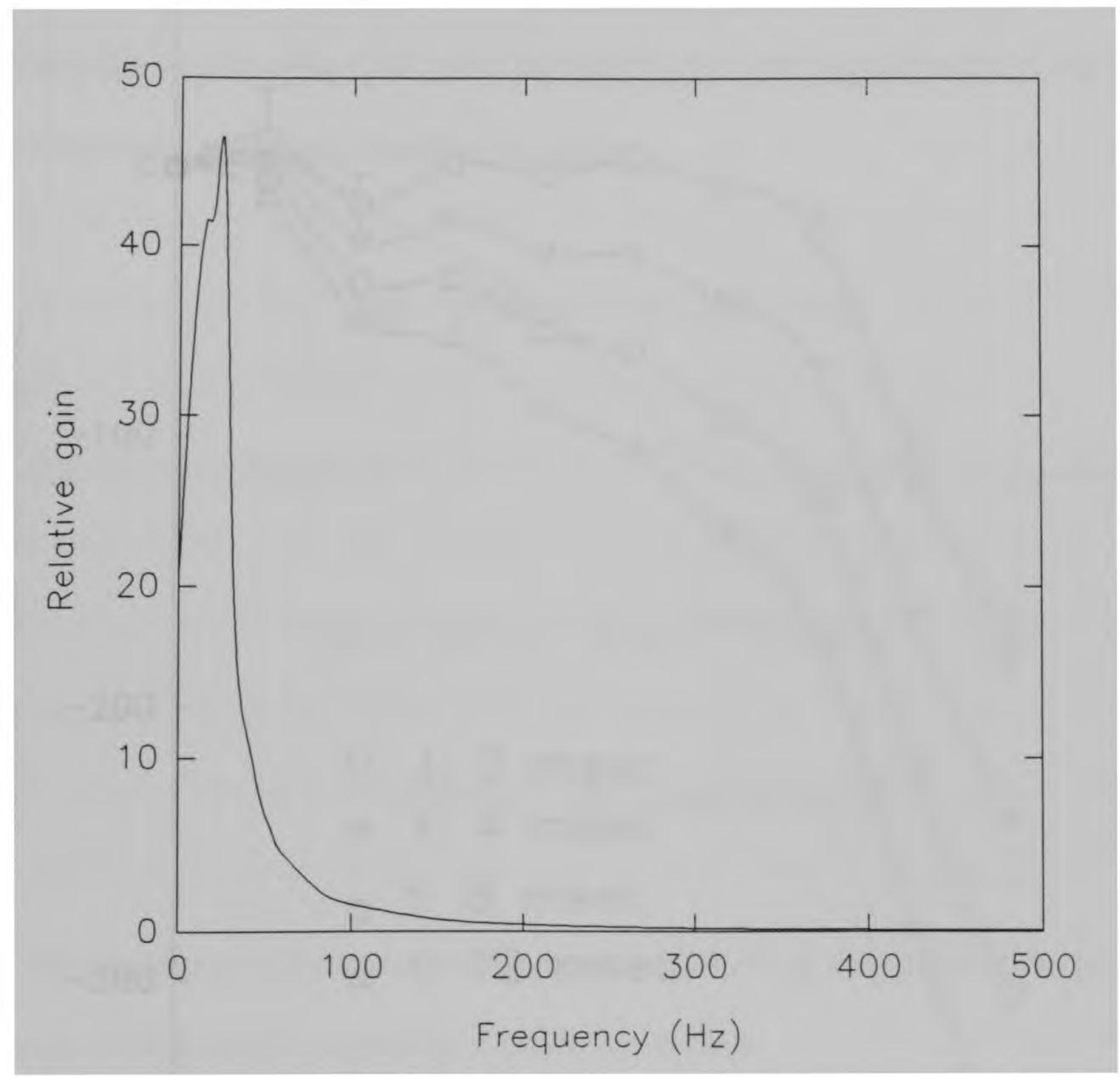

Figure 4.17 Relative gain curve

A resulting phase spectrum (subject R.A.B., $60 \%$ modulation) is shown in Fig. 4.18 (dotted line). Also shown are the measured phase spectra assuming different flash time delays (See Fig. 4.16). None of these matches the derived phase spectrum, particularly above $\sim 35 \mathrm{~Hz}$. It may therefore be concluded that the system does not exhibit minimum-phase characteristics. 


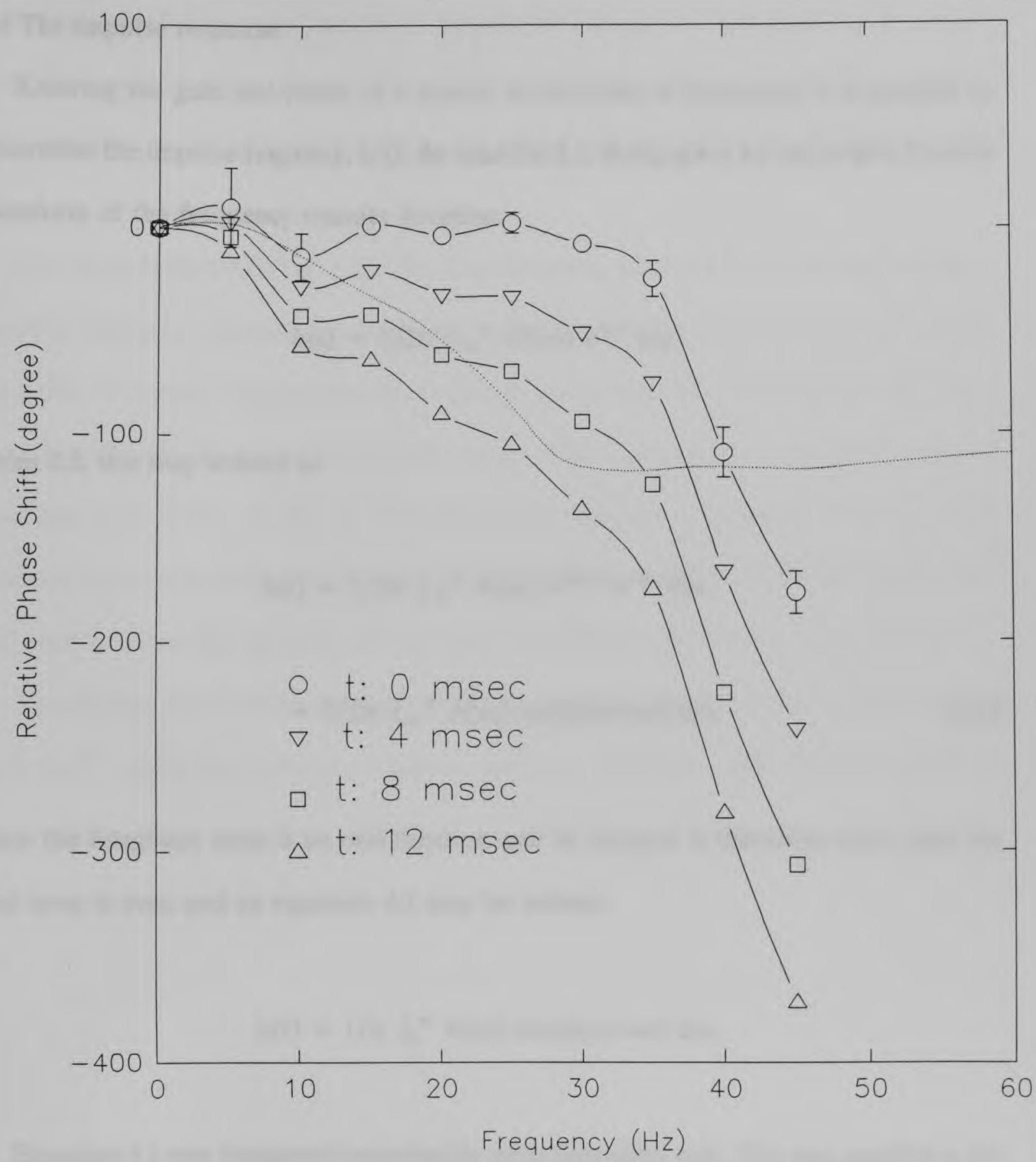

Figure 4.18 Phase shift spectrum 


\subsection{The impulse response}

Knowing the gain and phase of a system as functions of frequency, it is possible to determine the impulse response, $\mathrm{h}(\mathrm{t})$. As noted in 2.1, this is given by the inverse Fourier transform of the frequency transfer function:

$$
h(t)=1 / 2 \pi \int_{-\infty}^{\infty} G(i \omega) e^{-i \omega t} d \omega .
$$

From 2.3, this may written as

$$
\begin{aligned}
h(t) & =1 / 2 \pi \int_{-\infty}^{\infty} A(\omega) e^{i \phi(\omega)} e^{-i \omega t} d \omega . \\
& =1 / 2 \pi \int_{-\infty}^{\infty} A(\omega) \cos [\phi(\omega)-\omega t] d \omega,
\end{aligned}
$$

since the imaginary term is an odd function and its integral is therefore zero. Also the real term is even and so equation 4.1 may be written

$$
\mathrm{h}(\mathrm{t})=1 / \pi \int_{0}^{\infty} \mathrm{A}(\omega) \cos [\phi(\omega)-\omega \mathrm{t}] \mathrm{d} \omega .
$$

Equation 4.1 was integrated numerically using Simpson's rule. This was applied to the gain and phase spectra for subject R.A.B. (60\% modulation). A problem associated with numerical evaluation of a Fourier transform is that a finite number of Fourier components leads to a periodic function of time. It was therefore essential to choose a 
sufficiently small frequency interval to ensure that the period was significantly greater than the temporal extent of $h(t)$. This was found to be the case using a $0.1 \mathrm{~Hz}$ frequency interval which produced a period of $10 \mathrm{~s}$, compared with $\mathrm{h}(\mathrm{t})$ which extended over $\sim 200$ ms.

The result is shown in Fig. 4.19. The impulse response is seen to be clearly biphasic, possibly triphasic, although the oscillations above $\sim 50 \mathrm{~ms}$ may be essentially noise. Causality, of course, requires that the response be zero for $\mathrm{t}\langle 0$. In fact some oscillations (not shown) were also observed in this region. They may be the result of the lack of available data above $45 \mathrm{~Hz}$. In calculating the integral of equation $4.1, \mathrm{~A}(\omega)$ was assumed to be zero above this frequency. It should be noted that the higher frequencies will tend to affect the structure of $h(t)$ more significantly for shorter time intervals.

Nonetheless, the impulse response of Fig. 4.19 is remarkably similar to those derived by Kelly ${ }^{[27]}$, which also showed a triphasic response. They are produced in Fig. 4.20 for comparison. 


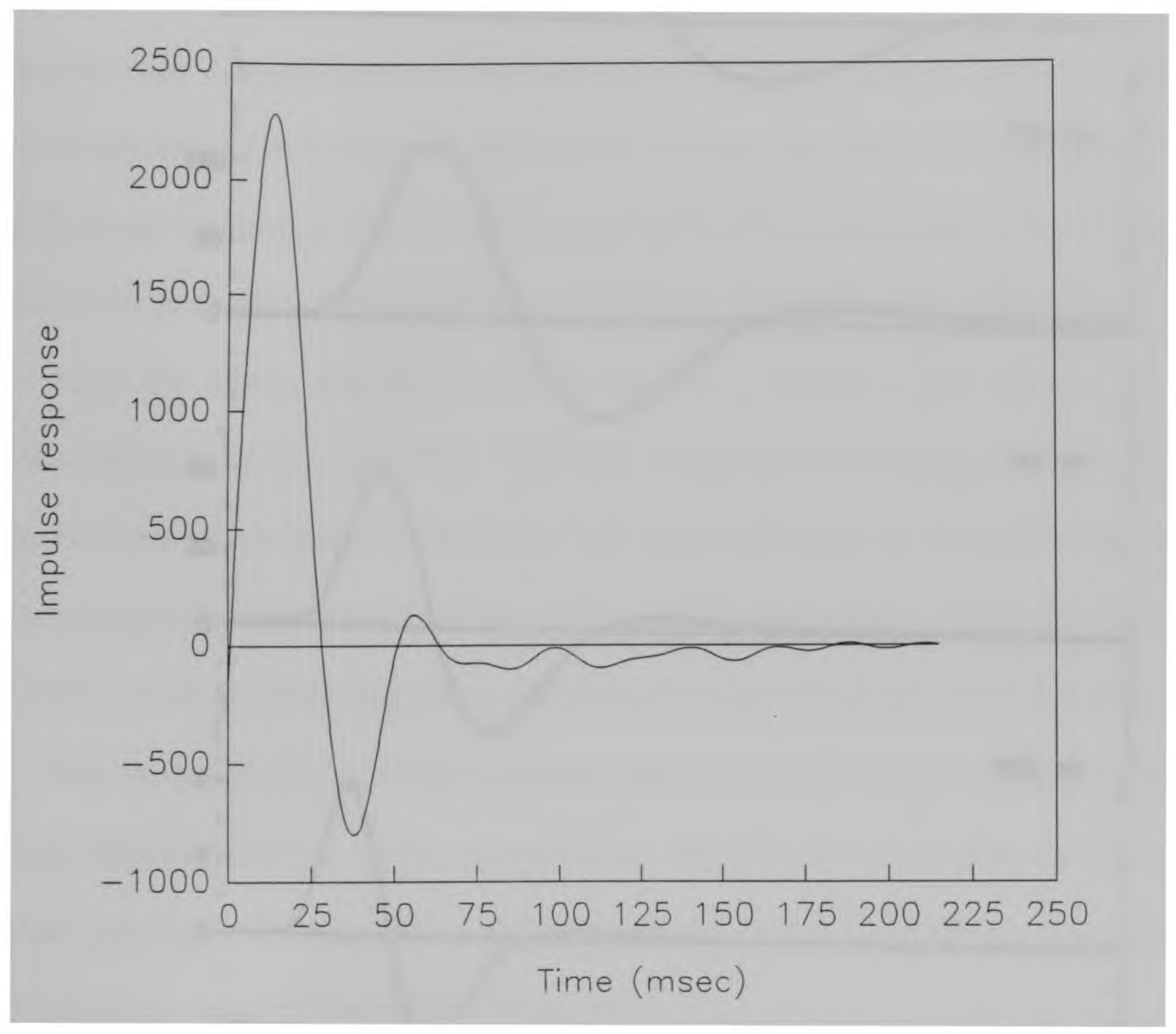

Figure 4.19 Impulse response 


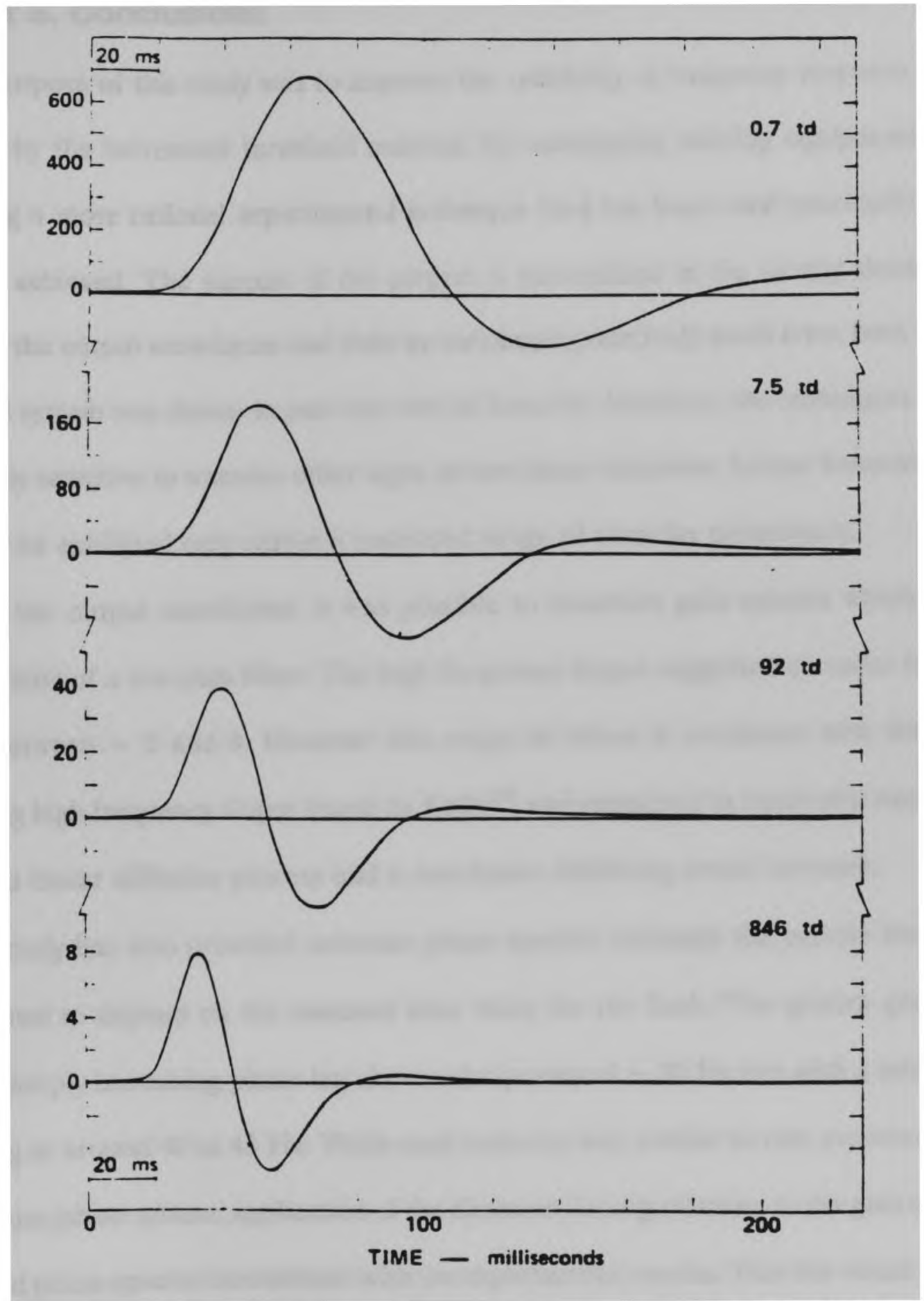

Figure 4.20 Impulse response of the two-stage model. (After [27]) 


\section{Chapter 5. Conclusions}

The purpose of this study was to improve the reliability of frequency response data obtained by the increment threshold method. By redesigning existing equipment and employing a more rational experimental technique than has been used previously, this goal was achieved. The success of the project is exemplified in the clearly sinusoidal nature of the output waveforms and their accompanying relatively small error bars. Thus the visual system was shown to pass one test of linearity. However, the techniques were sufficiently sensitive to uncover other signs of non-linear behavior. Linear behavior was shown to be exhibited only within a restricted range of stimulus parameters.

From the output waveforms, it was possible to construct gain spectra which were characteristic of a low-pass filter. The high frequency slopes suggested an order for the system between $\sim 5$ and 8 . However this range of values is consistant with the ever increasing high frequency slopes found by Kelly ${ }^{[27]}$ and explained in terms of a two-stage model - a linear diffusion process and a non-linear inhibiting neural network.

The study has also provided accurate phase spectra, although the precise form has been shown to depend on the assumed time delay for the flash. The spectra generally show a sharply increasing phase lag above a frequency of $\sim 30 \mathrm{~Hz}$ but with a minimum occurring at around 40 to $45 \mathrm{~Hz}$. While such behavior was similar to that expected from a minimum-phase system, application of the Kramers-Kronig relations to the gain spectra produced phase spectra inconsistent with the experimental results. That the visual system does not appear to be a minimum-phase system is not altogether surprising. As Victor ${ }^{[17]}$ 
pointed out, experiments on animals where gain and phase are measured often reveal the presence of non-minimum-phase transducers.

The reliability of the gain and phase measurements may be judged by the derived impulse response function which showed similarity to those obtained by others. ${ }^{[27]}$

What future work involving sinusoidal stimuli and the increment threshold method is warranted? One interesting modification would be to arrange for a constant amplitude output by suitably adjusting the input modulation at different frequencies. This may be compared with the present situation where the input amplitude was kept constant with frequency. The advantage of the former has been discussed by Stork ${ }^{[42]}$. Suppose that the portion of the system being investigated is linear, but it is followed by a non-linear stage. The proposed constant output method might then have advantages over the constant input method. For example, with the former method, the input to the non-linear stage would be of constant amplitude, regardless of frequency. An amplitude related nonlinearity would treat all frequencies equally and would not show up in the overall frequency response. On the other hand, a constant amplitude input to the linear stage would result in a frequency dependent input amplitude to the non-linear stage, and this could mask the linearity of the linear stage. Of course, if there is a non-linear stage followed by a linear stage, there is no advantage to either method.

Perhaps a bigger advantage of the constant output method is that it would allow for a more meaningful comparison with the extremely important C.F.F. results, such as Kelly's ${ }^{[27]}$, which relied on this technique, and on which the whole foundation of temporal processing is built. 


\section{References:}

[1] Ives, H. E., "Critical frequency relations in scotopic vision," J. Opt. Soc. Am. 6, 254 (1922)

[2] Ives, H. E., "A theory of intermittent vision," J. Opt. Soc. Am. and Rev. Sci. Instr. $6,343(1922)$

[3] Delange, H. D., "Experiments on flicker and some calculations on electrical analogue of the fovea systems," Physica 18, 935 (1952)

[4] Delange, H. D., "Relationship between critical flicker frequency and a set of low frequency characteristic of the eye," J. Opt. Soc. Am. 44, 380 (1954)

[5] Delange, H. D., "Thesis," Technical University, Delft, Holland. (1957)

[6] Delange, H. D., "Research into the dynamic nature of the human fovea-cortex systems with intermittent and modulated light. I. Attenuation characteristics with white and colored light," J. Opt. Soc. Am. 48, 777 (1958)

[7] Fuortes, M. G. F. and Hodgkin, A. L., "Change in time scale and sensitivity in the ommatidia of limulus," J. Physiol., 172, 239 (1964)

[8] Jones, R. C., "Electrophoresis apparatus," J. Wash. Acad. Science. 131, 100, (1957)

[9] Veringa, F., "On some properties of nonthreshold flicker" J. Opt. Soc. Am. 48, 500 (1958)

[10] Levinson, J., "Fusion of complex flicker" Science. 130, 919 (1959)

[11] Levinson, J., "Fusion of complex flicker II" Science. 131, 1438 (1960) 
[12] Forsyth, D. M., "Use of Fourier model in describing the fusion of complex visual stimuli" J. Opt. Soc. Am. 50, 337 (1960)

[13] Delange, H. D., "Eye's response at flicker fusion to square-wave modulation of a testfield surrounded by a large steady field of equal mean luminance," J. Opt. Soc. Am., 51, 415 (1961)

[14] Kelly, D. H., "Flicker fusion and harmonic analysis," J. Opt. Soc. Am. 55, 917 (1961)

[15] McTavish, J. L., "A study of temporal signal processing in the human visual system," Dissertation, University of the West Indies (1980)

[16] Stork, D. G., and Falk, D. S., "Temporal impulse response from flicker sensitivities," J. Opt. Sco. Am. A, 4, 1130 (1987)

[17] Victor, J. D., "Temporal impulse responses from flicker sensitivities: causality, linearity, and amplitude data do not determine phase," J. Opt. Soc. Am. A. 14, 1130 (1989)

[18] Bone, R.A., McTavish, John., Tang, Jun Ping., "Gain and phase characteristics of temporal processing determined by increment threshold" Invest. Ophthalmol. Vis. Sci., 32 (Suppl.), 852 (1991)

[19] Stiles, W. S., "Increment thresholds and the mechanism of color vision," Docum. Ophthal., 3, 138 (1949)

[20] Graham, C. H., "Sensation and perception in an objection psychology" Psychol. Rev., 65, 65 (1958)

[21] Crawford, B. H., "Visual adaptation in relation to brief condition stimuli," Pro. 
Roy. Soc. B. 283, 302 (1947)

[22] Boynton, R. M., Sturr, J. F. and Ikeda, M., "Study of flicker by increment threshold technique," J. Opt. Soc. Am. 51, 196 (1961)

[23] Boynton, R. M. and Siegfried, J., "Psychophysical estimates of no-responses to brief light flashes," J. Opt. Soc. Am. 52, 720 (1962)

[24] Bartley, S. H., "Vision - a study of its basis, " Hafner Publishing Company, New York (1963)

[25] Faulkner, E. A., "Introduction to the theory of linear systems" Printed in Great Britain by Willmer Brithers Limited, Birkenhead (1969)

[26] Nussenzeweig, H. M., "Casualty and dispersion relations," Academic, New York (1978)

[27] Kelly, D. H., "Theory of flicker and transient responses, I. uniform fields," J. Opt. Soc. Am. 61, 537 (1971)

[28] Kelly, D. H., "Flicker" Hb. Sensory Physiology, Vol. VII/4 Springer-Verlag, Berlin (1972)

[29] Kelly, D. H., "Diffusion model of linear flicke responses," J. Opt. Soc. Am., 59, $1665(1969)$

[30] Veringa, F., "Diffusion model of linear flicker responses," J. Opt. Soc. Am., 60, 285 (1970)

[31] Kelly, D. H., "Visual signal generator," Rev. Sci. Instr., 32, 50, (1960)

[32] Mason, S. J., and Zimmerman, H. J., "Electronic circuits, signals and systems," John Wiley \& Sons, Inc., New York (1960) 
[33] Davies, G., "Integral transforms and their applications," Springer-Verlag, New York, (1978)

[34] Guillemin, E. A., "Synthesis of passive networks," Wiley, New York (1957)

[35] Tang, J. P., "Increment threshold method in the study of the temporal response characteristic of the human visual system," Thesis, Florida International University, (1991)

[36] Pinter, R. B., "Sinusoidal and delta function responses of visual cells of the limulus eye," J. Gen. Physiol., 49, 565 (1966)

[37] Levinson, J., "One-stage model for visual temporal integration," J. Opt. Soc. Am., 56, $95(1966)$

[38] Matin, L., "Critical duration,the differential, luminance threshold, critical flicker frequency, and visual adaptation: a theoretical treatment," J. Opt. Soc. Am., 58, $404(1968)$

[39] Sperling, G. and Sondhi, M. M., "Model for visual luminance discrimination and flicker detection," J. Opt. Soc. Am., 58, 1133 (1968)

[40] Dagnelie, G., "Temporal impulse response from flicker sensitivities: practical considerations," J. Opt. Soc. Am. 9, 659, (1992)

[41] Westheimer, G., "The Maxwellian view," Vision Res., 6, 669, (1966)

[42] Stork, D. G., "Determination of symmetry and phase in human visual response functions: theory and experiments," Ph.D. dissertation, University of Maryland, College Park, Md., (1984) 